\title{
Homogenisation of Structural Breaks in the Global ESA CCI Soil Moisture Multi-Satellite Climate Data Record
}

\author{
Wolfgang Preimesberger, Tracy Scanlon, Chun-Hsu Su, Alexander Gruber, \\ and Wouter Dorigo
}

\begin{abstract}
The ESA CCI Soil Moisture COMBINED product is a more than $\mathbf{4 0}$ year long data record on global soil moisture for climate studies and applications. It merges soil moisture observations derived from multiple active and passive satellite remote sensing instruments in the microwave domain. Differences in sensor characteristics (such as frequency or polarisation) can cause structural breaks in the product, which are not completely removed during the merging process. These artificially caused discontinuities can adversely affect studies using the long-term data set. Here we compare three adjustment methods in terms of reducing the number of detected breaks in the soil moisture record. We investigate their impact on the data with multiple validation metrics. Their potential (negative) influence is examined by comparing trends in the data before and after homogenisation. We find that all three presented methods can reduce the number of detected breaks in ESA CCI SM. Differences between the methods mainly concern their ability to handle inhomogeneities in variance. Evaluation of the corrected data shows limited impact of homogenisation in terms of quantitative validation metrics. Changes in soil moisture trends due to removing breaks are found in some areas. We find that break correction overall improves the already rather homogeneous data set while preserving its climate describing characteristics. Quantile Category Matching is identified as the preferred method in terms of correcting breaks in ESA CCI SM.
\end{abstract}

Index Terms-Break detection, break correction, climate data record, homogeneity, microwave remote sensing, soil moisture

\section{INTRODUCTION}

A S part of the European Space Agency's (ESA) Climate Change Initiative (CCI), global long-term soil moisture (SM) data sets are derived from space-borne active and passive microwave remote sensing instruments [1]-[4]. Due to the limited operational period of single satellite systems, ESA CCI SM uses multiple SM products from ongoing and past remote sensing programs to create harmonised, merged, long-term data sets. As multiple different constellations of SM products from microwave sensors with varying properties and characteristics (active and passive) are combined, inhomogeneities (or structural breaks) at the transitions between the merged sensor periods (Fig. 1) may not be completely removed during the

W. Preimesberger (e-mail: wolfgang.preimesberger@geo.tuwien.ac.at), T. Scanlon and W. Dorigo are with the Department of Geodesy and Geoinformation, TU Wien, 1040 Vienna, Austria.

C.-H. Su is with the Bureau of Meteorology, Docklands, 3008 Victoria, Australia.

A. Gruber is with the Department of Earth and Environmental Sciences, KU Leuven, 3001 Heverlee, Belgium. dataset generation. Su et al. [5] found that breaks in ESA CCI SM mainly show as jumps in mean (sometimes in variance) between subsets of single time series when compared to a reference record. These breaks are originally caused by biases between the different SM products [6] and can partly remain (in strongly reduced form) throughout the scaling/merging scheme of ESA CCI SM. Structural breaks in climate data records can negatively influence the conclusion of scientific analyses, as they make data unrepresentative of the actual climate variability over time [7], [8]. The main concern is that inhomogeneities and discontinuities might be misinterpreted as natural occurrences and therefore affect the quality and conclusions of data applications. Especially for trend detection, inhomogeneities may conceal the true characteristics of the measurements. Su et al. [5] found evidence that ESA CCI SM trends at locations where inhomogeneities are detected match slightly worse with trends in reanalysis and insitu data than those at locations without a break.

The importance of detecting and correcting such (systematic) errors in any climate record has been highlighted by various other studies in the past [9]. Detection and homogenisation of structural breaks has been subject of many studies on terrestrial observations, mainly on measurements of temperature and precipitation. Potential reasons for inhomogeneities in these records were found due to urbanisation, station movement, or change in measurement sensor type [10]-[12]. Independent of the measured variable, studies that test the homogeneity of climate records suggest that no long-term measurement data set is free of inhomogeneities.

More and more data from Earth observation satellites are becoming available and an increasing number of disciplines is using them. Available data records are combined to overcome the temporal limitations of single sensor products [13] and therefore the detection of inhomogeneities in these records is becoming the topic of research (e.g. Pinzon et al. [14] on NDVI, Brinckmann et al. [15] for solar irradiance data, Schroeder et al. [16] for satellite water vapour). However, correcting detected breaks in combined satellite data sets is still a major challenge. SM records in general have received little to no consideration in this regard. This is most likely because most of them have covered relatively short time periods until now. Methods for detection and adjustment depend on the type of climate record. Temperature and SM are continuous variables, whereas for example precipitation is discontinuous [17]. SM is limited by the soil saturation. Correction methods 
may also depend on the type of shifts that are found in the data set, e.g. breaks in mean and variance. Some studies also adapt correction methods to regional circumstances, to better take into account - for example - local climate or weather [18], [19].

In this study we compare three different homogenisation methods. They aim to adjust inhomogeneities in the global ESA CCI SM v04.5 COMBINED multi-satellite product by matching homogeneous sub-periods that were not sufficiently homogenised during the initial merging process [1], [4]. We compare long-term trends in the data before and after correcting detected breaks to understand the potential impact of inhomogeneities in the data. The aim is to reduce the number of detected breaks and optimise the adjustment process in terms of minimising error metrics with respect to (mostly) independent reference datasets.

In Section II the candidate (ESA CCI SM) and reference data used in the homogenisation and evaluation process are described in more detail. The methods for the detection of breaks are shortly reviewed in Section III. Section III also looks at developing, adapting and implementing three separate break correction methods within a satellite SM specific framework. It further contains short descriptions of the common methodology to evaluate the corrections. Section IV compares the results for different methods in terms of the reduction of breaks in ESA CCI SM. The impact of adjustment on error metrics and long term trends in the data set is also described there.

\section{DATASETS AND PREPROCESSING}

\section{A. ESA CCI SM}

ESA CCI SM is a long term climate data record (CDR) on global SM, based on multiple satellite remote sensing products (as indicated by sensor names in Fig. 1 for v04.5 of the COMBINED product). SM measurements from an (increasing) number of satellite-based active (scatterometers, Fig. 1 blue names) and passive (radiometer, Fig. 1 red names) microwave observation systems are used in the merging process. ESA CCI SM is available as three separate products, which contain only active, only passive and both types of measurements (the here used COMBINED product of ESA CCI SM v04.5, referred to as ESA CCI SM from here on) [1], [4]. Weights for merging available sensor products are derived from signal-to-noise ratio (SNR) estimates from Triple Collocation (TC) analysis [3]. Homogenisation of the single products is achieved via scaling by matching cumulative distribution functions (CDF) with respect to SM simulations from the Global Land Data Assimilation System (GLDAS) Noah v2.1 [20] land surface model as the common reference data set. The most recent version of ESA CCI SM spans over a period of over 40 years (1978-10-26 to 2018-12-31).

ESA CCI SM observations are representative of water in the top layer of the soil (first few centimetres, depending on the radiometric properties of the merged sensors at any given time) and expressed in volumetric units $\left(\mathrm{m}^{3} \mathrm{~m}^{-3}\right)$. The data is organised on a global quarter degree grid. Observation flags as well as error estimates for single observations are provided with the SM measurements. We use only the unflagged ("good") observations of ESA CCI SM in this study. The original daily images were converted into a time series format with varying temporal coverage, depending on the number and orbits of the sensor platforms in use. From the nine sensor transition dates as shown in Fig. 1 (excluding the start and end date), seven are considered in this study, excluding October 2011 and October 2007 to avoid sensor sub-periods that are shorter than one year. This results in a total of eight (assumed) homogeneous sensor sub-periods (SSPs) in between the potential breaks. The ESA CCI SM product is released together with a static mask of densely vegetated areas, which covers about $6 \%$ of all global land points (excluding Antarctica). SM retrievals from satellite observations are unreliable in these (tropical rainforest) areas [21]. Therefore they are excluded in all results and areas are marked in most maps with dark green colour.

\section{B. MERRA2}

In this study we use relative methods to detect and correct breaks. This means that for testing and adjusting the homogeneity of single candidate observation series we rely on (homogeneous) reference observations for comparison in nonparametric statistical tests [5] and also for characterisation of detected breaks for subsequent adjustment. The candidate and reference datasets must be similarly influenced by climate and weather to distinguish between artificial and natural shifts. Assuming that the reference is homogeneous, artificial breaks (that are introduced by a change in the measurement setup) would only appear in the candidate. Naturally caused changes would be represented in both datasets [22]. Finding a suitable reference for a candidate is a fundamental part of all relative break testing/adjustment methods. Most studies prefer using in situ measurements to analyse the homogeneity of other nearby stations over reanalysis data as they are considered to contain less (and better documented) breaks [23]. In situ SM observation sites are, however, limited in coverage and generally span shorter time periods than satellite observations [24]. Therefore, the use of in situ reference data for homogenising (multi-)satellite observations is not feasible in our case and a global long-term reference product is needed. Land surface models replicate the interactions of climate processes on Earth and therefore assimilate observations that indirectly affect the simulated SM state (e.g. precipitation). Satellite SM observations are not directly assimilated in most models, which are therefore as independent as possible from the ESA CCI SM data set. The use of reanalysis reference data for detection and adjustment of breaks in climate variables is a common practice in homogenisation studies for cases where in situ measurements are not sufficiently available (e.g AzorinMolina et al. [25] on wind speed observations). A single series from the reference data set can be used directly - the nearest neighbour is usually considered to show the most similar naturally caused deviations - or multiple nearby references can be combined to create a benchmark for the candidate [26]. As spatial inconsistencies in model SM simulations are assumed to be small, the creation of merged reference series (e.g. from 
neighbouring pixels) is considered not to be necessary within this study.

In this study, we used the National Aeronautics and Space Administration (NASA) Global Modelling and Assimilation Office's (GMAO) Modern-Era Retrospective analysis for Research and Applications 2 (MERRA2) data set [27], [28]. MERRA2 provides hourly soil water simulations starting with the beginning of the year 1980 until present. It therefore covers a similar time period as ESA CCI SM. We select SM simulations from the (single) nearest grid point in the MERRA2 reanalysis reference data set to act as a the reference for each according candidate. The data is computed on a global $0.5^{\circ} \times 0.625^{\circ}$ grid. The original hourly fields were temporally resampled to daily averages to match the temporal sampling of ESA CCI SM.

By using MERRA2 SM, we follow $\mathrm{Su}$ et al. [5], who found SM from the MERRA-Land model [29] skillful in terms of global detection of inhomogeneities. Both MERRA2 and MERRA-Land use observation-based precipitation forcing for creating SM simulations [30]. Model SM is therefore assumed to contain less inhomogeneities compared to ESA CCI SM, especially in areas with precipitation measurements. We use the "Water Surface Layer" (referred to by the official short name SFMC) variable of MERRA2 (in $\mathrm{m}^{3} \mathrm{~m}^{-3}$ ). It is representative of SM in the top ten centimetres of the soil and therefore comparable to ESA CCI SM observations. A bias between the two is expected, because of the different model scales and computational differences of MERRA2 compared to the scaling reference used in the production of ESA CCI SM (the GLDAS Noah v2.1). This bias is accounted for by matching the CDF of the reference series to that of the candidate series at each grid cell [31], [32]. A predefined set of percentiles $(0,5,10,30,50,70,90,95,100)$ with a linear interpolation between them is used.

\section{ECMWF reanalysis}

We use two reanalysis products from the European Centre for Medium-Range Weather Forecasts (ECMWF) for validation [33], i.e. simulations from the recently released ERA5 reanalysis [34], [35], which is the successor to the ERAInterim reanalysis [36], as well as from the according ERAInterim/Land product [37]. ERA5 provides global, sub-daily simulations of variables for land, atmosphere and ocean waves. 6-hourly images (starting at 0:00 UTC) with a spatial resolution of $0.25^{\circ} \times 0.25^{\circ}$ of the topmost $(0-7 \mathrm{~cm})$ of four available soil water layers - the "Volumetric soil water layer 1" (SWVL1) variable between 1979 and 2018 - were temporally resampled (arithmetic mean of 6-hourly simulations) to match the daily resolution of ESA CCI SM. ERA-Interim/Land uses near-surface meteorological fields of ERA-Interim together with precipitation adjustments to force the HTESSEL land surface model, leading to improvements in terms of land water simulations compared to the ERA-Interim reanalysis. A temporally extended version of the data set was used, in which the "Volumetric Soil Moisture of Layer 1" variable is available in the period from 1979 until 2015 with a horizontal resolution of $\sim 80 \mathrm{~km}$. Similar as for ERA5, ERA-Interim/Land simulations were temporally resampled to daily averages.

\section{METHODS}

\section{A. Break Detection}

To detect breaks in ESA CCI SM, we follow Su et al. [5]. This method uses relative tests with model reanalysis reference data on SM difference series $(\Delta S M)$ between candidate observations from ESA CCI SM and the corresponding reference simulations from MERRA2. Difference series do not include naturally caused variations (that are not breaks) between SSPs - since they should exist in both datasets. The null hypothesis for break detection therefore is, that there are no significant differences in the first two moments of distributions of $\Delta S M$ before and after the tested sensor transition date. In ESA CCI SM the potential break dates are defined by variations in the sensor products that are combined (the SSPs shown in Fig. 1) and therefore known beforehand. SSPs form the minimum input sets for break detection and correction. As will be shown later, input data for testing and correction are not restricted to SSPs but can be extended across negatively tested (i.e. homogeneous) transition dates. We formulate pairs of adjacent SSP difference series as described in (1) and (2) as the input sets for statistical testing.

$$
\begin{aligned}
& \Delta S M_{S S P_{i, 0}}=C A N_{S S P_{i, 0}}-R E F_{S S P_{i, 0}} \\
& \Delta S M_{S S P_{i, 1}}=C A N_{S S P_{i, 1}}-R E F_{S S P_{i, 1}}
\end{aligned}
$$

For each sub-period $S S P_{i, j}, i \in[0, . ., 7]$ is the identifier of the tested transition date, with $i=0$ as the latest date of potentially introducing a break. $j$ marks whether the respective period is before $(j=1)$ or after $(j=0)$ the tested transition date that separates the two sub-periods (compare to Fig. 2b). For testing, common daily candidate $(C A N)$ and reference $(R E F)$ values were jointly temporally resampled as monthly means with a required daily coverage per month of $>30 \%$ [5]. Therefore $>9$ days per month are required to calculate the arithmetic mean that is then representative for the month, otherwise no value is assigned. Monthly averages are chosen as the input to reduce noise and outliers in each SSP, which could make detection of mean and variance breaks unreliable.

Breaks were detected by relative, nonparametric, statistical testing [7], [38], following two methods that were found applicable to the ESA CCI SM v02.2 COMBINED data set by $\mathrm{Su}$ et al. [5]. First we use the nonparametric Wilcoxon rank-sum test (WK) [39] for detection of shifts in ranked, tie-corrected [40] difference values, which corresponds to an assumed mean break in the candidate. Second the nonparametric FlignerKilleen test (FK) [41] detects relative shifts in the variance of two differences series. Su et al. [5] found both methods skilful in terms of odds ratio (incorrect versus correct detection of an inhomogeneity) and Hanssen-Kuipers discriminant (skill of separating detected inhomogeneities from homogeneities) when comparing results from relative testing with model reference data to testing with in situ measurements. The resampled observations are again scaled before calculating $\Delta S M$ within the SSP pairs using linear regression scaling to reduce common short term biases that may exist across the tested transition date. Relative biases (i.e. breaks that should 
be detected) in mean and variance between the two periods are not affected by this scaling choice.

The input observations used in break testing need to comply with two predefined statistical requirements. 1) The number of monthly values in $S S P_{i, 0}$ and $S S P_{i, 1}$ is sufficiently large $(N>10)$. 2) Spearman's $\mathrm{R}\left(R_{S P}\right)$ between the candidate and reference is greater than 0.5 and significant $(p<0.05)$ across the combined sub-periods (joined period $S S P_{i, 0}$ with $\left.S S P_{i, 1}\right)$. If any of these two preconditions fails, the respective transition date is not tested.

We identify three possible types of sensor transition dates in ESA CCI SM in each location. 1) Homogeneous (negatively tested) transition dates: Both null hypotheses (by WK and FK) cannot be rejected. 2) Inhomogeneous (positively tested) transition dates: A statistically significant break in mean, variance (or both) is detected. 3) Untested transition dates: Both tests are not applied because one (or both) of the prerequisites is not fulfilled.

\section{B. Break Correction}

1) Procedure Overview: To homogenise detected breaks in ESA CCI SM, an iterative adjustment approach is implemented, starting from the most recent SSP. For each candidate series we start by detecting breaks between SSPs as described in Section III-A and as schematically shown in Fig. 2 b.

Then - based on these (initial) test results - breaks are quantified by comparing extended SSPs, which are referred to as model sub-periods (MSPs). This means that the sensor periods before and after detected breaks are expanded across negatively tested (homogeneous) sensor transition dates (compare e.g. $M S P_{1,1}$ in Fig. 2c) or successfully removed ones (during previous correction iterations, as indicated by the dashed horizontal line part of $M S P_{2,0}$ in Fig. 2c). Extension is not performed across detected breaks and untestable dates (compare e.g. $M S P_{1,1}$ respectively $M S P_{1,0}$ in Fig. 2c). This allows to increase the number of input values for correction which makes it more robust. To ensure that the period extension does not affect the test result, break detection is repeated with values in the MSPs (instead of the SSPs) before attempting to correct any re-detected breaks. If the break is not detected again using the extended period, it is not corrected. As an additional requirement to the re-detection, correction is only attempted if the candidate and reference correlate sufficiently with Pearson's correlation coefficient $R_{P}>0.3$. This is done on top of the previous evaluations for testing, to make prerequisites for correction even more restrictive (step I. in Fig. 3). $R_{P}$ is checked for both MSPs around the break (for the LMP and QCM methods, described in Sections III-B2 and III-B4), respectively only for $M S P_{i, 0}$ (for the HOM method, described in Section III-B3).

In addition to finding MSPs, the initial break detection is also used to define adjustment sub-periods (ASPs, see Fig. 2c) for each iteration of correction and between detected breaks. As not all sensor transition dates can be tested, it is necessary to separate the input values used for quantifying a break (MSPs) from values that corrections are applied to (ASP). On the one hand this is done to avoid that derived corrections are distorted by inhomogeneous observations, which could be the case if $M S P_{i, 1}$ included untestable subperiods that are potentially inhomogeneous. On the other hand this avoids the accumulation of previously corrected shifts at earlier, untestable transition dates. Notably, $M S P_{i, 1}$ and $A S P_{i}$ are equal in cases where there is no untestable transition date between two detected breaks (e.g. $M S P_{1,1}=A S P_{1}$ in Fig. 2c). Applying correction factors to ASPs, that were found between MSPs in cases when $M S P_{i, 1} \neq A S P_{i}$ therefore assumes that values that are in $A S P_{i}$ but not in $M S P_{i, 1}$ are shifted accordingly. Then not only are the MSPs matched, but also untested or homogeneous transitions would remain unchanged (as will be shown later this is not always the case). This correction procedure (step II a. and II b. in Fig. 3) is evaluated to some extent in a third iteration of break detection - after adjustment - again using the MSPs as inputs (step III. in Fig. 3). We evaluate: 1) Whether a break was completely removed and 2) whether the relative bias between $A S P_{i}$ and $M S P_{i, 0}$ (i.e. before and after the break) has decreased.

If one of the two checks after adjustment fails, the correction is reverted and the values from before adjusting the current subperiod are restored (step IV a. in Fig. 3). Equations (3)(5) describe the second (bias) constraint between the adjusted (ADJ) and uncorrected (CAN) candidate with respect to the reference $(\mathrm{REF})$ data in the according periods.

$$
\Delta B I A S_{i, \text { adjusted }} \leq \Delta B I A S_{i, \text { unadjusted }}
$$

with

$$
\begin{array}{r}
\Delta B I A S_{i, \text { adjusted }}=\mid B I A S(A D J, R E F)_{A S P_{i}}- \\
B I A S(C A N, R E F)_{M S P_{i, 0}} \mid
\end{array}
$$

and

$$
\begin{array}{r}
\Delta B I A S_{i, \text { unadjusted }}=\mid B I A S(C A N, R E F)_{A S P_{i}}- \\
B I A S(C A N, R E F)_{M S P_{i, 0}} \mid
\end{array}
$$

As indicated by (3)-(5) and Fig. 2c, we aim to relatively fit the period before a break to the homogeneous period after the break. The most recent homogeneous sub-period $\left(M S P_{0,0}\right)$ is referred to as the base period, as iterative adjustment progresses towards the beginning of the candidate series. Values in the base period are never changed. The most recent homogeneous sub-period is chosen as the base period for the following reasons: First, because later periods of ESA CCI SM show higher temporal coverage compared to earlier periods due to the larger number of merged sensor products (compare to SI Fig. 5). Second, because the quality of used sensors has improved over time, resulting in the most accurate observations for later periods. Lastly, because compared to other sensor periods, with more than six years in length this is a relatively long (assumed) homogeneous period without changes in the merged sensor products.

Fig. 3 shows the complete previously described processing cycle for correcting multiple breaks at a single location and the three (interchangeable) principles (LMP, HOM and QCM in step II. of Fig. 3) to quantify the size of detected inhomogeneities and subsequently remove them. The goal is to identify one approach that not only reduces breaks in ESA 
CCI SM and improves its quality, but simultaneously preserves the (not break-related) characteristics.

To explain and compare the three different adjustment principles in an example case in the following sections, values for ESA CCI SM and MERRA2 in the homogeneous period between 2010-01-12 and 2018-12-31 for a single point in North Louisiana (Lat: $32.875^{\circ}$, Lon: $-91.625^{\circ}$ ) are used. ESA CCI SM values before 2012-07-01 have been distorted by an arbitrary multiplicative factor of 1.1 and an additive bias of $+0.05 \mathrm{~m}^{3} \mathrm{~m}^{-3}$ to create a synthetic mean-break of known size (detected by WK, also illustrated in SI Figs. 1, 2 and 3).

2) Method 1: Linear Regression Model Pair Matching $(L M P)$ : For the first method we assume that detected relative breaks in each candidate can be quantified by differences in the parameters of two linear regression models [42]. The first model is found between the candidate and reference in $M S P_{i, 0}$. The second one accordingly within $M S P_{i, 1}$. Monthly resampled candidate and reference observations (as in Section III-A) are used as inputs to model the linear relationship $Y=X \cdot[\alpha, \beta]^{T}+\epsilon$ with candidate observations $(\mathrm{Y})$, the design matrix $(\mathrm{X})$, the model intercept $(\alpha)$ and slope $(\beta) . \epsilon$ describes the (minimised) residuals between the leastsquares predicted and the observed values. A break (in mean or variance) in the candidate across $M S P_{i, 0}$ and $M S P_{i, 1}$ leads to differences in the model coefficients $\alpha_{M S P_{i, 0}}$ and $\alpha_{M S P_{i, 1}}$, respectively $\beta_{M S P_{i, 0}}$ and $\beta_{M S P_{i, 1}}$ of the two sub-periods. Discrepancies in $\alpha$ and $\beta$ represent additive respectively multiplicative relative biases.

To reduce the impact of outliers on the models, in addition to using monthly values we exclude the $5 \%$ of observation pairs with the largest corresponding $|\Delta S M|$. It is assumed that single outliers are not caused by relative breaks between the compared periods as breaks affect the whole period.

Fig. 4 shows the linear regression models for the two MSPs around the introduced break in the example series in North Louisiana. The calculated model coefficients for the biased observations in $M S P_{1,0}$ were found with $\alpha=0.15$ and $\beta=$ 0.75 from 29 observations (Fig. 4a) and for $M S P_{0,0}$ with $\alpha=0.10$ and $\beta=0.68$ from 69 observations in this period (Fig. 4b). The introduced break is therefore mainly represented as a difference in the $\alpha$ coefficient in the example case.

As discrepancies in the model coefficients represent the size of the detected break they can be used to find required corrections as described in (6)-(8). $C A N$ describes the monthly values, that are also used for finding the regression models. $C A N_{M S P_{i, 1}}^{\text {correction }}$ contains monthly corrections for each candidate value before the introduction date of the detected break.

$$
C A N_{M S P_{i, 1}}^{\text {correction }}=\left(r \cdot C A N_{M S P_{i, 1}}+c\right)-C A N_{M S P_{i, 1}}
$$

with

$$
r=\left(\frac{\beta_{M S P_{i, 0}}}{\beta_{M S P_{i, 1}}}\right)
$$

and

$$
c=\left(\alpha_{M S P_{i, 1}}-r \cdot \alpha_{M S P_{i, 0}}\right)
$$

To apply the corrections ( $\left.C A N_{M S P_{i, 1}}^{\text {correction }}\right)$ to observations in the extended period $A S P_{i}$, we follow Vincent et al. [19] and find one average correction for each month of $C A N_{M S P_{i, 1}}^{\text {correction }}$ (i.e. the arithmetic mean of all corrections for January, for February, and so on). We then multiply the so found vector with the tridiagonal A-matrix (9) from Sheng et al. [43] to find a mid-month "target" value (at day 15) of each month. Linear interpolation between these "target" values leads to daily corrections that are - for each month - on average equal to the original monthly corrections.

$$
A=\left(\begin{array}{ccccc}
7 / 8 & 1 / 8 & & & \\
1 / 8 & 6 / 8 & 1 / 8 & & \\
& \ddots & \ddots & \ddots & \\
& & 1 / 8 & 6 / 8 & 1 / 8 \\
& & & 1 / 8 & 7 / 8
\end{array}\right)
$$

These adjustments are then repeatedly applied (added) to the original inputs in the period to adjust $\left(A S P_{i}\right)$, until the next break (or the beginning of the observation series). This way we adjust observations in $M S P_{i, 1}$ and theoretically also match the remaining values in $A S P_{i}$ accordingly without introducing (undetectable) relative differences between them.

3) Method 2: Higher Order Moments Adjustment (HOM): This homogenisation method was originally proposed by Della-Marta et al. [44] and successfully used to correct inhomogeneities in in situ temperature observations. It makes use of a quadratic regression model from the candidate and reference after a break to predict and correct observations before the break. Polynomial regressions allow considering potential nonlinearities, which may appear between satellite and reanalysis data, when quantifying breaks. They may be caused for example by (too) high reanalysis SM auto-correlation or varying discrepancies between model and satellite observations (e.g. for different seasons due to the unreliability of satellite SM estimates under frozen soil conditions).

We start by fitting a second order polynomial regression model between the ESA CCI SM candidate and MERRA2 reference series in the MSP after the break (a linear model is used in cases where $R_{P}>0.8$ with $p<0.05$ between the candidate and reference in $\left.M S P_{i, 0}\right)$. Again monthly resampled and filtered values (as in Section III-B2) are used as inputs. The nonlinear model from candidate and reference time series for the base period $\left(M S P_{0,0}\right)$ is shown in Fig. 5b.

Together with the reference data in $M S P_{i, 1}$ the model is then used to create (homogeneous) predictions of the candidate. We find adjustments for the candidate observations based on these predictions: We use a theory of L-moments approach [45] to fit either a Normal, a Pearson Type Three, a Generalised Normal or a Generalised Extreme Value distribution to the candidate in the MSP after the break (compare SI Fig. 4). A Kolmogorov-Smirnoff test (KS) [46] is used to compare the ensemble of possible theoretical CDFs to the candidate observations and predictions. The best fitting distribution is selected by minimising the maximum deviation (the KS statistics) between fitted theoretical and empirical CDFs.

The so found CDF for the candidate after the break is used to calculate quantiles for the observations $\left(C A N_{M S P_{i, 1}}\right)$ 
and predictions $\left(P R E D_{M S P_{i, 1}}\right)$ before the break, respectively the observations in $A S P_{i}$. We then fit a Locally Weighted Scatterplot Smoothing (LOWESS) function [47] [48] to the SM differences (residuals) between the observations and predictions $\left(\triangle S M=C A N_{M S P_{i, 1}}-P R E D_{M S P_{i, 1}}\right)$ as shown in Fig. 6 for the demonstration case. LOWESS uses multiple linear estimates within local subsets of the residuals to fit a smooth polynomial. The factor $\alpha$ allows control over the smoothing by increasing/decreasing the subset size used for estimating the local regressions to combine. A fraction of $\alpha=0.6$ was chosen to fit a curve, that varies only little and represents the distinct adjustment of each quantile. These corrections are then applied (added) to all candidate observations in $A S P_{i}$ to relatively fit ESA CCI SM values before the break to observations in the MSP after the break with respect to changes in MERRA2.

4) Method 3: Quantile Category Matching adjustment (QCM): This method was proposed by Wang et al. [49], who adjusted breaks in (de-trended) precipitation series (absolute method) and adapted by e.g. Vincent et al. [50] to adjust breaks in temperature series using nearby in situ reference observations (relative method). It builds on the assumption that adjustments for a detected break can be derived by detecting differences in a number of (median) empirical cumulative frequencies $(\triangle E C F)$ of various quantile categories (QCs) between the candidate and the reference before and after the transition date. Each category contains observations over an equal range of quantiles. Differences are found by comparison of QC means of adjacent homogeneous sub-periods $\left(M S P_{i, 0}\right.$ and $\left.M S P_{i, 1}\right)$. Relative differences between the candidate and the reference across the detected break then represent the size of the break for each QC separately.

We start with a maximum of four (quartile) categories because ESA CCI SM sensor periods can be short (compare Fig. 1) and include a limited range of theoretically possible observation values. Reasons for this can be for example low climate variability, geophysical restraints or inherited limits from the CCI scaling reference (GLDAS Noah) [1]. We find quantiles by sorting (ranking) data in ascending order by the candidate subset and find for each (unique) candidate observation the normalised Cumulative Frequency (CF) in both MSPs around a detected break. We divide the ranked observations of each MSP into the selected number of categories, so that each category spans over an equal range of CFs (again by candidate). For each $Q C_{i} \in[1, \ldots, Q C]$ with $Q C \leq 4$ in each MSP, we find the median $\mathrm{CF}$ of its according $\mathrm{CF}$ range and find the mean of the differences between the candidate and the reference in this category $(\triangle E C F)$ as described in (10) and (11).

$$
\begin{aligned}
& \Delta E C F\left(\frac{Q C_{i}-0.5}{Q C}\right)_{M S P_{i, 0}}= \\
& \operatorname{mean}\left(C A N_{M S P_{i, 0}, Q C_{i}}-R E F_{M S P_{i, 0}, Q C_{i}}\right) \\
& \Delta E C F\left(\frac{Q C_{i}-0.5}{Q C}\right)_{M S P_{i, 1}}= \\
& \operatorname{mean}\left(C A N_{M S P_{i, 1}, Q C_{i}}-R E F_{M S P_{i, 1}, Q C_{i}}\right)
\end{aligned}
$$

$\triangle E C F$ for $M S P_{0,0}$ and $M S P_{0,1}$ for the example break are shown in Fig. 7a and $7 \mathrm{~b}$ where the dots are the differences between the median $\mathrm{CF}$ of the candidate and reference. The median $\mathrm{CF}$ represents the frequency range of each category (indicated by the bars).

In cases where the number of categories is too high (i.e. one or more category does not include any observations), we reduce QC by one until each category contains observations or only one category containing all observations for each MSP is left.

We then calculate the category differences between the $\triangle E C F$ of the two MSPs as described in (12).

$$
\begin{aligned}
& A\left(\frac{Q C_{i}-0.5}{Q C}\right)=\Delta E C F_{M S P_{i, 0}}\left(\frac{Q C_{i}-0.5}{Q C}\right)- \\
& \Delta E C F_{M S P_{i, 1}}\left(\frac{Q C_{i}-0.5}{Q C}\right)
\end{aligned}
$$

where $A$ are the corrections to fit the median $\mathrm{CF}$ of the candidate of the period before the break relatively to changes in the reference. To ensure a smooth transition across the categories, we fit a cubic spline to the median CFs, with $A(0)$ and $A(1)$ being equal to the first respectively last category difference value as shown in Fig. 7c. Notably, when the total number of categories $Q C=1$, all values before and after the break represent one group and adjustment results in a (constant) relative mean matching. To apply the adjustments to the candidate observations, we first find the ECF of all values to adjust $\left(A S P_{i}\right)$ and apply adjustments to them, that were calculated as described in (12). Therefore we shift the extended period according to corrections from the MSPs.

The blue bars in Fig. 7a show the differences in the quantile categories between the adjusted candidate and the reference in the MSP before the break. As adjustments were found based on the extended period $A S P_{0}, \triangle E C F_{0,1}$ after correction does not exactly match $\triangle E C F_{0,0}$ (in $7 \mathrm{~b}$ ), but is almost equal and differences are much smaller than before adjustment.

\section{Evaluation}

We evaluate and compare the fidelity of the described homogenisation approaches by using quantitative error metrics. In-depth validation of ESA CCI SM has been the subject of numerous studies [51], [52]. Our goal is to assess the impact of the three adjustment methods on the original data set. Validation is performed with respect to the ERA5 reanalysis as it can be considered mostly independent from the satellite SM observations and the MERRA2 reference product used in the adjustment process. We compute: bias (difference in means), root-mean-squaredifference (RMSD), unbiased root-mean-square-difference (ubRMSD), Pearson's and Spearman's correlation $\left(R_{P}\right.$ and $R_{S}$ ) as well as the mean-square error (MSE) and the residual sum of squares (RSS). Before calculation of the ubRMSD, ESA CCI SM datasets were scaled to ERA5 by matching their mean and standard deviation [32]. Biases between ESA CCI SM and ERA5 are expected to be dominated by discrepancies between GLDAS Noah (which is used in 
the production of ESA CCI SM) and ERA5. Error metrics are calculated for the single SSPs from Fig. 1 as well as for the full period. Intercomparison is performed only for locations that were adjusted in any sub-period and with all three methods. Therefore a total of 41518 points was used. As ERA5 is available in quarter degree resolution, validation of quasi-collocated grid cells (a constant offset between the cell origins was accounted for) can be performed.

We investigate the impact of adjustment methods on long term trends in ESA CCI SM by comparing changes over time between the unadjusted and adjusted versions and multiple reference products (ERA5, ERA-Interim/Land, MERRA2). The impact of adjustment methods on data set trends is of special interest, as ESA CCI SM aims to provide a data record for long term climate assessments. We follow the approach as described by Dorigo et al. [53] and Albergel et al. [54] and calculate Theil-Sen trend estimates [55] of seasonal averages together with a nonparametric Mann-Kendall test [56]-[58] to detect significant SM increase or decrease $(p<0.05)$ independent from how observations are distributed. Theil-Sen estimates provide linear fitting of the underlying observations that are robust to outliers due to using the median of multiple slope estimates. Only values after 1991-08-05 are used because of the consistency of underlying data sources. Linear regression based scaling [32] was applied to all datasets before calculation of slopes with the unadjusted observations acting as the scaling reference. Trends were calculated over all observations starting in 1991 as well as for pairs of subsequent SSPs after 1991 as these were the temporal basis for the applied correction.

\section{RESUlts AND Discussion}

\section{A. Global break detection and correction}

Fig. 8 shows location and types of detected breaks for the sensor transitions at 2012-07-01 (Fig. 8 left column) and 200206-19 (Fig. 8 right column) before and after adjustment with the three described methods. The first transition date in 2012 contains the last SSP of the data set (base period) and therefore represents the first iteration of adjustment performed on the candidate data. These results are not influenced by any previous correction iterations and should be easiest to reproduce. Therefore they best represent direct differences between the correction methodologies. As the sensor transition at 2011-1005 was excluded from the correction process (to avoid subperiods that are shorter than one year) potential breaks caused by changes in the sensor constellation from this date could affect the 2012 test results as they are included in $S S P_{0,1}$. The sensor transition in 2002 contains the largest number of detected breaks in the original data set (of both types) with respect to a relatively low testing coverage. Table I shows the detailed test statistics on the number of detected breaks and covered points for all seven considered sensor transition dates, before ("original") and after correcting detected breaks in ESA CCI SM with the respective method ("LMP", "HOM" and "QCM"). HOM uses mostly quadratic models (in $66 \%$ to $86 \%$ of concurrent cases, compare to SI Fig. 14). 1987-07-09 is excluded from the table as no testing is possible.
Figs. 8a and $8 \mathrm{~b}$ show the initial test results of the original SSPs in the unadjusted data set. For all tested transition dates the number of detected breaks in mean is 5-7 times larger than the number of variance breaks. The highest number of breaks in mean and most simultaneous (mean and variance) breaks are detected for the sensor transition in 2002-06-19 (29.13\% respectively $3.19 \%$, relative to the number of tested points). 1991-08-05 shows the highest relative coverage of variance breaks with $4.40 \%$. The least breaks are detected for the sensor transition in 2010-01-15. As three of the four sensors used after 2010 were already part of the merged data set in the preceding SSP, this indicates that the homogeneity of the data set is affected more if more sensor products are replaced or complemented by others and less if the sensor constellation remains similar. An overall decrease in the absolute number of tested points ("Test Coverage") towards earlier periods of ESA CCI SM can be observed. This is mainly due to the smaller number of available sensors, the observation frequency and therefore the data density in these periods but also due to slightly decreasing correlation with MERRA2. This leads to the prerequisites for testing not being met and therefore no testing being possible for the first sensor transition in 198707-09. The number of tested ESA CCI SM points for later periods increases from $\sim 20 \%$ in 1991 up to more than $50 \%$ in 2010 and 2012.

Figs. 8c-8h show results of a separate test run after correction with the three respective methods (compare to the statistics in Table I). The original SSPs without any extension (same as for the unadjusted data in $8 \mathrm{a}$ and $8 \mathrm{~b}$ ) were used. It can be seen that the number of detected breaks is significantly reduced by all three correction methods. Overall, the results for all three methods are very similar in terms of coverage and number and location of (un)removed breaks. The number of breaks in mean detected by WK is reduced by $\sim 70-80 \%$ compared to the original data. Repeated testing after QCM shows the lowest number of detected breaks (of both types) after adjustment for all tested dates compared to the other methods. All methods perform better in terms of reducing breaks in mean compared to removing variance breaks. Variance breaks are reduced by $20-30 \%$ by the QCM method for all dates, but LMP and HOM show for three sensor transitions even a minor increase in FK-positive points (red numbers in Table I). This can be the case as some breaks that were initially detected by both tests (mean and variance) got partly removed during earlier iterations of correction. This is due to the iterative correction approach, where one iteration might not only correct the break at its respective transition date (between MSPs) but also at the sensor transition date of the previous break, i.e. at the beginning of the adjusted period $A S P_{i}$. This explanation is supported by the fact that the number of concurrent mean and variance breaks ("Both") is reduced by all methods significantly, but again most for QCM. An increase in detected (only) variance breaks is therefore not necessarily an indicator of data degradation.

The remaining breaks that were detected after adjustment are either for points where preconditions were not met (and hence no adjustment was attempted) or where the adjusted values were discarded (as a bias was introduced or as a break 
was still detected after correction).

\section{B. Analysis of intermediate results}

In this section we compare some of the intermediate results of (global) break correction in more detail. Plots here only show a (representative) subset of the global results to visualise the (small) differences between the methods. The chosen region (grasslands/savannas) contains a large amount of breaks in mean and variance around the masked rainforest area. Fig. 9 shows the according subset of global break detection for the sensor transition in 1998-01-01. Fig. 9a shows results of the prerequisite checks before initial testing. Areas marked in blue were actually tested, leading to the detected homogeneities and breaks shown in Fig. 9b. Based on these results, MSPs and ASPs for adjustment were created by extending the original sensor periods. Adjustment methods are then applied, but only for points where also testing with MSPs detected a break. Comparing Figs. $9 \mathrm{~b}$ and $9 \mathrm{c}$ shows that this is the case for most detected breaks. This indicates that the use of extended homogeneous sub-periods for adjustment is a viable option to increase the data size for correction without changes in the detection of a break or (less important) its type.

Fig. 10 shows intermediate test results during correction as well as remaining breaks after correction for the same date. The left column shows detected breaks between MSPs after attempting to correct inhomogeneities as found in $9 \mathrm{c}$ with the three methods (LMP in line 1, HOM in line 2, and QCM in line 3). These tests represent the status of the adjusted candidate before deciding whether to discard or accept the adjusted values. It can be seen that all three methods manage to reduce breaks in mean. For QCM a small number and for HOM a slightly larger number of breaks in mean is still detected afterwards. It can also be seen in the left column that the number of breaks in variance increases for all methods (least for QCM) compared to Fig. 9c. This shows that correction methods overall achieve to adjust the shifts in mean, but simultaneously often introduce new ones in variance instead, and hence change the type of the break instead of removing it. For LMP this indicates that the differences in the parameters of the two linear models are in fact a representation of relative mean differences, but matching observations based on slope and intercept often fails to maintain the homogeneity in variances for daily observations. Similar results can be observed for HOM (Fig. 10d). This indicates that the LOWESS fitted quantile differences between the observed and predicted candidate values include the searched correction in mean but often also affect the variance. This could be due to differences in the distributions of the candidate in the used sub-periods and/or due to how corrections are derived from interpolation of the residuals (smoothing factor $\alpha$ ). QCM finally shows the lowest number of variance breaks in this regard. This indicates that even a small number of quantile categories $(Q C \leq 4)$ leads to a better performance in terms of restoring or maintaining the homogeneity in variance compared to the other two methods, albeit not in all cases.

The middle column in Fig. 10 compares adjustment output evaluations for the three methods. The three respective classes contain only points that were attempted to be adjusted (according to the left column). 1) "Accepted" contains points where correction was attempted and accepted (i.e. no break was detected anymore afterwards, output evaluations passed). The adjusted values are then stored. 2) "Break" indicates points, where after three repetitions of adjustment a break is still detected (the break type might have changed). In these cases the corrected values are rejected and discarded and the unadjusted observations within the (unsuccessfully) adjusted sub-period are restored. 3) " $>\Delta$ Bias" indicates that the difference in bias between candidate and reference in the adjusted period $A S P_{i}$ and the reference period $M S P_{i, 0}$ was not reduced. These corrections are then rejected as well and the original values are restored.

Finally, the right column in Fig. 10 shows test results for the adjusted products. They bring into context the coverage of adjusted and unadjusted areas for each method to a separate test run with the homogenised data set, again with the (original, unextended) SSPs. As expected the patterns of remaining breaks are similar to the evaluations in the middle column (unadjusted breaks should be detected again), but differences are expected due to the different time periods used.

Table II shows statistics for the global adjustment coverage (compare to the middle column of Fig. 10) for the three methods, i.e. the number of points where adjusted values were accepted, correction was attempted but adjusted values were discarded ("> $\Delta$ Bias" and "Break") or correction was not attempted ("UnCorr."). QCM shows the largest number of accepted adjustments, globally about $10 \%$ more than the other two methods, for which $60-70 \%$ of corrections were accepted. This corresponds with the lowest number of detected breaks after adjustment with QCM as mentioned in the discussion of Fig. 8. As expected HOM has the smallest number of points where adjustment was not attempted due to too low correlation ("UnCorr."), as for this method only the MSP after the break is evaluated, whereas for LMP and QCM $R_{P}$ of both periods $M S P_{i, 0}$ and $M S P_{i, 1}$ - is checked separately. For up to $15 \%$ of detected breaks adjusted values are discarded due to a relative increase in bias. This can be the case if $A S P_{i} \neq M S P_{i, 0}$ and could for example be due to an undetected break in the adjusted period if corrections that aim to match the MSPs are then applied to all values. The number of points for which the corrections were not accepted for this reason is similar across the three methods. Especially the transitions in 1991 and 2010 show a higher number of these cases. QCM introduces biases slightly more often than the other two methods, but this is due to more points being evaluated in this regard as less (variance) breaks remain after correction.

\section{Impact of adjustment}

1) Longest Homogeneous Period: Fig. 11 shows the length on the longest homogeneous period, i.e. the maximum period in ESA CCI SM over which no break was detected (compare to $\mathrm{Su}$ et al. [5]). Untested sensor transitions are treated in the same way as positively tested ones (not homogeneous). Fig. 12 shows changes in the length of the longest homogeneous period after correction with the three respective methods. Due 
to the large number of removed breaks - especially in regions in Central Africa, Australia and South America - an increase in homogeneous period length, leading to up to 30 years long periods, can be observed. Northern regions as well as SouthEast Asia and Europe are less or not affected due to the overall low number of tested points there. Differences in the longest homogeneous periods between the methods can only be found for regions where the performance of adjustment methods differs, i.e. mostly in regions where variance breaks were detected. QCM (Fig. 12c) shows the longest homogeneous periods e.g. in Central Africa, North America or Australia.

2) Comparison against independent reanalysis data: Global comparison of validation metrics between the unadjusted and adjusted versions of ESA CCI SM against ERA5 SWVL1 shows only small differences between the methods. For the full period (between 1979 and 2018) we find that $R_{P}$ of 41518 adjusted points increased in $55.54 \%$ of cases by up to 0.055 (for the 99th percentile) and decreased for the remaining $44.46 \%$ by down to -0.048 (for the first percentile of the differences). Slightly better results are found for QCM, where $R_{P}$ for $59.95 \%$ of points increased by up to 0.057 . For HOM we find a decrease in $R_{P}$ for $56.11 \%$ of pixels, which indicates a slightly worse performance than the other two methods. A decrease in $R_{P}$ can be observed mostly in vegetated areas - around the masked areas. As expected, we find similar results for ubRMSD. Further, QCM shows the best performance of the three methods with an overall decrease in ubRMSD with areas in Central Africa and South-America showing small increases (maps can be found in the SI Figs. 3538). The same applies to the other metrics that were calculated, although MSE and RMSD are found to decrease in most of the before mentioned vegetated areas (e.g. in South-America). Differences in the metrics for single SSPs are found to be generally slightly more distinctive than for the full period. For example in $S_{S} P_{4} \Delta R_{P}$ ranges between -0.14 (HOM) and 0.08 for (LMP) and in $S S P_{1} \triangle R M S D$ is between -0.04 $m^{3} m^{-3}$ and $0.03 m^{3} m^{-3}$. Compared to the other methods, QCM shows the largest improvements during SSP validation with up to $70 \%$ of pixels showing a better correspondence with ERA5 after correction. Notably we detect a slight decrease in Spearman's R $\left(R_{S}\right)$ after LMP and HOM with respect to ERA5 (up to -0.3 for single points in some of the earlier SSPs) whereas changes for QCM are closer to zero or even positive. For a detailed representation of differences in the metrics for the three compared methods, we refer to SI Table I as well as the box plots and maps in SI Figs. 23-34. Overall we find that reducing the number of breaks in ESA CCI SM with the described methods has little impact on the temporal correspondence with ERA5. This indicates that the corrected relative biases within the product are small compared to the overall deviations. QCM shows the best overall performance in terms of the described metrics (shown for ubRMSD in Fig. 13).

3) Trend analysis: Fig. 14 shows the correspondence in (significant) SM trends in ESA CCI SM before and after QCM adjustment. The respective actual, significant trends over the period 1991-2018 in the original and QCM adjusted ESA CCI SM data are shown in Figs. 15a and 15b (see SI Fig. 39 for the according plots for LMP and HOM, and SI Fig. 43 for the actual difference values). For comparison also trends in three reanalysis variables are shown: SWVL1 from ERAInterim/Land in Fig. 15c (1991-2015) and from ERA5 in Fig. $15 \mathrm{~d}$ as well as for the reference data used in the adjustment process - MERRA2 SFMC - in Fig. 15e. Differences between ESA CCI SM and reanalyses trends are evident from Fig. 15. Trends match better for the three sub-periods in SI Figs. 40-42 than for the full period.

Most significant trends are preserved during adjustment (grey areas in Fig. 14) or no significant trends are introduced (white areas in Fig. 14). There are nearly no locations in Fig. 14 where a significant drying trend changed into a significant wetting trend or vice versa. This indicates that - even though a large number of inhomogeneities was removed - the original ESA CCI SM was influenced only little by the removed breaks and that most corrected breaks were small. This also applies to the sub-period trends. As $\mathrm{Su}$ et al. [5] pointed out, trends across inhomogeneities can be similar to those in reanalysis reference data, explaining why correction shows only limited change in this regard. Overall this strengthens the confidence in the merging scheme of ESA CCI SM and its viability for creating a data set for long term studies. How much single (extreme) values or noise are affected by the correction procedure is not evident from this analysis.

There are however some areas where trends that were not significant before adjustment became significant (light red and light blue areas in Fig. 14), or where significant trends are not detected anymore after adjustment (orange and turquoise areas in Fig. 14). All three adjustment methods generally slightly increase the number of detected significant trends in the data set $(\mathrm{LMP}+6 \%, \mathrm{HOM}+5 \%$, QCM $+7 \%)$. Fig. 14 highlights some areas where QCM introduced significant trends. Trends from the reference data set (Fig. 15e) are adopted in most of these cases. Area (A) in Fig. 14 shows a negative trend after correction and likewise do areas (B) and (C) (compare to Figs. 15a and 15b). The latter two are (partly) aligned with the negative trends in MERRA2. In general, trends for areas around the dense vegetation mask seem to be affected more by the correction process. This could again be due to discrepancies between the MERRA2 model and ESA CCI SM in these areas (as was shown in Section IV-C2 for the ERA5 reanalysis). The positive trends in area (D) are mostly in line with the models. In the Eastern parts of (D) more significant drying trends than in the original ESA CCI SM are detected. Areas (E), (F) and (G) show the opposite effect, as trends after correction in these areas are mostly positive. For $(\mathrm{E})$ and $(\mathrm{F})$ the trends are in line with MERRA2, for $(G)$ the trends after adjustment correspond also to ERA-Interim/Land (Fig. 15c).

4) Impact of reference data: Relative testing to model reference data is affected by the homogeneity of the reference data set. MERRA2 was chosen as the reference to detect and correct breaks as it is independent from data used in ESA CCI SM and as Su et al. [5] found that the gaugebased precipitation forcing in MERRA products provides homogeneous enough SM values to act as the (sole) reference. Other reanalysis data are available and can be used in place of MERRA2. Differences between the SM variables of these 
products is expected with regard to the underlying models, the assimilation fields and forcing data. Yet we found only small differences when using ERA5 in place of MERRA2 in a separate iteration of the QCM correction procedure. SI Figs. 45-47 compare the detected breaks before and after correction with ERA5 as the reference to the original results with MERRA2 and the according SM trends. Some differences for the later sensor periods of ESA CCI SM between detected breaks with MERRA2 and ERA5 as the reference source are found (e.g. Australia, South America), while for earlier periods the results are similar. This might be due to the availability of actual SM observations in later periods (satellite SM is assimilated in ERA5 [34]) and/or the distribution if rain gauge measurements (used in MERRA2). Therefore the reference might affect the homogenisation result (i.e. the adjusted values), while the correction itself was found to perform similar (i.e. in number of removed breaks) for ERA5 and MERRA2. Also trends in ESA CCI SM are found to remain similar independent of the chosen reference. Completely homogeneous reference data is not available. As an alternative using multiple reference sources for detection/correction and/or for (independent) evaluation could be an option. Correction could then only be attempted at locations where an agreement between multiple reference data sets is found.

\section{CONCLUSiON}

In this study we adapted three methods to reduce structural breaks in the ESA CCI SM v04.5 COMBINED data set. Break detection was implemented in line with the preceding study by $\mathrm{Su}$ et al. [5]. Potential breaks in mean and variance as well as homogeneous and untestable transitions between sensor period subsets were detected and reduced by up to $80 \%$ with three relative (independent) approaches. All three methods depend on the use of reanalysis reference data, which was considered to be homogeneous, although it might not be [23]. Within the common framework that the methods are embedded into, extended homogeneous sub-periods from initial testing were used for quantifying detected breaks and for correction. Period extension allows applying corrections to observations between detected breaks whilst deriving them only from observations within homogeneous sub-periods. This compromise is necessary as not all sensor transitions of ESA CCI SM can be tested reliably. Repeated break detection after correction reveals that a large number of breaks (in mean) is removed by all methods. Of the three methods, Quantile Category Matching reduces the number of mean breaks the most and is also the only method to reliably reduce the number of detected variance breaks. We therefore consider it the preferred method. Validation of the datasets after correction shows only little change in terms of bias, (ub)RMSD and correlation with respect to independent reanalysis reference data. In some areas break correction altered trends in ESA CCI SM. These alterations where mostly in line with the reference data set used for correction. Especially for grid cells in the Northern Hemisphere trends remained mostly unaffected. Overall we consider the limited changes in terms of error metrics and trends a confirmation of the merging approach of ESA CCI SM, as it indicates that the removed breaks were small. The impact of adjustment can be larger for spatial or temporal subsets of the data record.

Potential ways to improve the correction methods include considering different noise levels between sub-periods for quantifying breaks and during evaluation. To improve the correction of variance breaks less restrictions in terms of variability of derived corrections (e.g with a higher number of quantile categories or a different smoothing factor for interpolation) can be explored. The framework presented here could make use of methods for joint correction [59] instead of the iterative approach that is currently implemented and make use of multiple locations (neighbourhoods) to detect and quantify breaks more reliably. The implemented iterative approach applies corrections to observations between initially detected breaks. As nonparametric tests are used to detect breaks and not all breaks can be removed, this could - to some extend - still lead to increasing the size of detected yet not corrected breaks.

Break detection and correction should be seen as an integral part during the creation and validation of multi-sensor based climate records. This holds true for satellite-based data sets as it does for terrestrial records. Choosing suitable reference data to find breaks in satellite records is especially difficult due to the spatial and temporal coverage needed. Therefore absolute methods for break correction are of particular interest as satellite observations are known to contain features that are not represented in state of the art land surface models, such as irrigation [60].

Python code for break detection and correction from this study is publicly available on GitHub at https://github.com/ TUW-GEO/pybreaks .

\section{ACKNOWLEDGMENTS}

The development of the ESA CCI products has been supported by ESA's Climate Change Initiative for Soil Moisture (Contract No. 4000104814/11/I-NB and 4000112226/14/INB). The ESA CCI SM data can be accessed after registration at https://www.esa-soilmoisture-cci.org/. The Copernicus Climate Change Service (C3S) soil moisture product is funded by the Copernicus Climate Change Service implemented by ECMWF through C3S 312b Lot 7 Soil Moisture service. This work was supported by the QA4SM project (https: //qa4sm.eodc.eu/), funded by the Austrian Space Applications Programme (FFG). Generated using C3S Information 2019 (ERA5, ERA-Interim/Land), accessible at https://cds.climate. copernicus.eu/. MERRA2 is available at https://gmao.gsfc. nasa.gov. Copyright notice: (c) ESA Climate Change Initiative - Land Cover project 2017. 


\section{REFERENCES}

[1] W. Dorigo, W. Wagner, C. Albergel, F. Albrecht, G. Balsamo, L. Brocca, D. Chung, M. Ertl, M. Forkel, A. Gruber, E. Haas, P. D. Hamer, M. Hirschi, J. Ikonen, R. de Jeu, R. Kidd, W. Lahoz, Y. Y. Liu, D. Miralles, T. Mistelbauer, N. Nicolai-Shaw, R. Parinussa, C. Pratola, C. Reimer, R. van der Schalie, S. I. Seneviratne, T. Smolander, and P. Lecomte, "Esa cci soil moisture for improved earth system understanding: State-of-the art and future directions," Remote Sensing of Environment, vol. 203, pp. 185-215, 2017, Earth Observation of Essential Climate Variables. DOI: 10.1016/j.rse.2017.07.001.

[2] Y. Liu, W. Dorigo, R. Parinussa, R. de Jeu, W. Wagner, M. McCabe, J. Evans, and A. van Dijk, "Trend-preserving blending of passive and active microwave soil moisture retrievals," Remote Sensing of Environment, vol. 123, pp. 280-297, 2012. DOI: 10.1016/j.rse.2012. 03.014 .

[3] A. Gruber, W. Dorigo, W. Crow, and W. Wagner, "Triple collocationbased merging of satellite soil moisture retrievals," IEEE Transactions on Geoscience and Remote Sensing, vol. 55, no. 12, pp. 6780-6792, Dec. 2017. DOI: 10.1109/TGRS.2017.2734070.

[4] A. Gruber, T. Scanlon, R. van der Schalie, W. Wagner, and W. Dorigo, "Evolution of the esa cci soil moisture climate data records and their underlying merging methodology," Earth System Science Data Discussions, vol. 2019, pp. 1-37, 2019. DOI: 10.5194/essd-2019-21.

[5] C.-H. Su, D. Ryu, W. Dorigo, S. Zwieback, A. Gruber, C. Albergel, R. H. Reichle, and W. Wagner, "Homogeneity of a global multisatellite soil moisture climate data record," Geophysical Research Letters, vol. 43, no. 21, pp. 11, 245-11, 252, 2016. DOI: 10.1002/ 2016 GL070458.

[6] R. H. Reichle and R. D. Koster, "Bias reduction in short records of satellite soil moisture," Geophysical Research Letters, vol. 31, no. 19, 2004. DOI: $10.1029 / 2004 G L 020938$.

[7] T. C. Peterson, D. R. Easterling, T. R. Karl, P. Groisman, N. Nicholls, N. Plummer, S. Torok, I. Auer, R. Boehm, D. Gullett, L. Vincent, R. Heino, H. Tuomenvirta, O. Mestre, T. Szentimrey, J. Salinger, E. J. Førland, I. Hanssen-Bauer, H. Alexandersson, P. Jones, and D. Parker, "Homogeneity adjustments of in situ atmospheric climate data: A review," International Journal of Climatology, vol. 18, no. 13, pp. 1493-1517, 1998. DOI: 10.1002/(SICI)1097-0088(19981115)18: 13〈1493::AID-JOC329〉3.0.CO;2-T.

[8] V. Conrad and L. W. Pollak, "Methods in climatology.," Quarterly Journal of the Royal Meteorological Society, vol. 77, no. 332, pp. 328-330, 1951. DOI: $10.1002 /$ qj.49707733228.

[9] T. R. Karl and C. N. Williams, "An approach to adjusting climatological time series for discontinuous inhomogeneities," Journal of Climate and Applied Meteorology, vol. 26, no. 12, pp. 1744-1763, 1987. DOI: $10.1175 / 1520-0450(1987) 026\langle 1744$ :AATACT $\rangle 2.0 . C O ; 2$.

[10] B. C. Trewin and A. C. F. Trevitt, "The development of composite temperature records," International Journal of Climatology, vol. 16, no. 11, pp. 1227-1242, 1996. DOI: 10.1002/(SICI)1097-0088(199611) 16:11<1227::AID-JOC82 $>3.0 . C O ; 2-P$.

[11] X. L. Wang, Q. H. Wen, and Y. Wu, "Penalized maximal t test for detecting undocumented mean change in climate data series," Journal of Applied Meteorology and Climatology, vol. 46, no. 6, pp. 916-931, 2007. DOI: 10.1175/JAM2504.1.

[12] J. R. Lanzante, "Resistant, robust and non-parametric techniques for the analysis of climate data: Theory and examples, including applications to historical radiosonde station data," International Journal of Climatology, vol. 16, no. 11, pp. 1197-1226, 1996. DOI: 10.1002/ (SICI)1097-0088(199611)16:11 <1197::AID-JOC89〉3.0.CO;2-L.

[13] R. Hollmann, C. J. Merchant, R. Saunders, C. Downy, M. Buchwitz, A. Cazenave, E. Chuvieco, P. Defourny, G. de Leeuw, R. Forsberg, T. Holzer-Popp, F. Paul, S. Sandven, S. Sathyendranath, M. van Roozendael, and W. Wagner, "The esa climate change initiative: Satellite data records for essential climate variables," Bulletin of the American Meteorological Society, vol. 94, no. 10, pp. 1541-1552, 2013. DOI: 10.1175/BAMS-D-11-00254.1.

[14] J. E. Pinzon and C. J. Tucker, "A non-stationary 1981-2012 avhrr ndvi3g time series," Remote Sensing, vol. 6, no. 8, pp. 6929-6960, 2014. DOI: $10.3390 /$ rs6086929.

[15] S. Brinckmann, J. Trentmann, and B. Ahrens, "Homogeneity analysis of the $\mathrm{cm}$ saf surface solar irradiance dataset derived from geostationary satellite observations," Remote Sensing, vol. 6, no. 1, pp. 352-378, 2014. DOI: $10.3390 / \mathrm{rs} 6010352$

[16] M. Schroeder, M. Lockhoff, J. M. Forsythe, H. Q. Cronk, T. H. Vonder Haar, and R. Bennartz, "The gewex water vapor assessment: Results from intercomparison, trend, and homogeneity analysis of total column water vapor," Journal of Applied Meteorology and Climatology, vol. 55, no. 7, pp. 1633-1649, 2016. DOI: 10.1175/ JAMC-D-15-0304.1.

[17] M. Hulme, "Estimating global changes in precipitation," Weather vol. 50, no. 2, pp. 34-42, 1995. DOI: 10.1002/j.1477-8696.1995. tb06074.x.

[18] H. Tuomenvirta, "Homogeneity adjustments of temperature and precipitation series-finnish and nordic data," International Journal of Climatology, vol. 21, no. 4, pp. 495-506, 2001. DOI: 10.1002/joc.616.

[19] L. A. Vincent, X. Zhang, B. R. Bonsal, and W. D. Hogg, "Homogenization of daily temperatures over canada," Journal of Climate, vol. 15 , no. 11, pp. 1322-1334, 2002. DOI: 10.1175/1520-0442(2002) 015 $\langle 1322:$ HODTOC $\rangle 2.0 . C O ; 2$.

[20] M. Rodell, P. R. Houser, U. Jambor, J. Gottschalck, K. Mitchell, C.-J. Meng, K. Arsenault, B. Cosgrove, J. Radakovich, M. Bosilovich, J. K. Entin, J. P. Walker, D. Lohmann, and D. Toll, "The global land data assimilation system," Bulletin of the American Meteorological Society, vol. 85, no. 3, pp. 381-394, 2004. DOI: 10.1175/BAMS-853-381.

[21] T. J. Jackson, T. J. Schmugge, and J. R. Wang, "Passive microwave sensing of soil moisture under vegetation canopies," Water Resources Research, vol. 18, no. 4, pp. 1137-1142, 1982. DOI: $10.1029 /$ WR018i004p01137.

[22] C. T. Peterson and D. Easterling, "Creation of homogeneous composite climatological reference series," International Journal of Climatology, vol. 14, pp. 671-679, Jul. 1994. DOI: 10.1002/joc.3370140606.

[23] A. Sterl, "On the (in)homogeneity of reanalysis products," Journal of Climate, vol. 17, no. 19, pp. 3866-3873, 2004. DOI: 10.1175/15200442(2004)017〈3866:OTIORP $\rangle$ 2.0.CO;2.

[24] W. Dorigo, W. Wagner, R. Hohensinn, S. Hahn, C. Paulik, A. Xaver, A. Gruber, M. Drusch, S. Mecklenburg, P. van Oevelen, A. Robock, and T. Jackson, "The international soil moisture network: A data hosting facility for global in situ soil moisture measurements," Hydrology and Earth System Sciences, vol. 15, no. 5, pp. 1675-1698, 2011. DOI: 10.5194/hess-15-1675-2011.

[25] C. Azorin-Molina, S. M. Vicente-Serrano, T. R. McVicar, S. Jerez, A. Sanchez-Lorenzo, J.-I. López-Moreno, J. Revuelto, R. M. Trigo, J. A. Lopez-Bustins, and F. Espírito-Santo, "Homogenization and assessment of observed near-surface wind speed trends over spain and portugal, 1961-2011," Journal of Climate, vol. 27, no. 10, pp. 3692-3712, 2014. DOI: 10.1175/JCLI-D-13-00652.1.

[26] K. W. Potter, "Illustration of a new test for detecting a shift in mean in precipitation series," Monthly Weather Review, vol. 109, no. 9, pp. 2040-2045, 1981. DOI: $10.1175 / 1520-0493(1981) 109\langle 2040$ : IOANTF $>2.0 . \mathrm{CO} ; 2$

[27] R. Gelaro, W. McCarty, M. J. Suárez, R. Todling, A. Molod, L. Takacs, C. A. Randles, A. Darmenov, M. G. Bosilovich, R. Reichle, et al., "The modern-era retrospective analysis for research and applications, version 2 (merra-2)," Journal of Climate, vol. 30, no. 14, pp. 5419-5454, 2017.

[28] GMAO. (2015). Merra-2 tavg1_2d_lnd_nx: 2d 1-hourly timeaveraged single-level assimilation, land surface diagnostics v5.12.4 English, [Online]. Available: https://disc.gsfc.nasa.gov.

[29] R. H. Reichle, R. D. Koster, G. J. M. De Lannoy, B. A. Forman, Q. Liu, S. P. P. Mahanama, and A. Touré, "Assessment and enhancement of merra land surface hydrology estimates," Journal of Climate, vol. 24 , no. 24 , pp. $6322-6338$, 2011. DOI: $10.1175 /$ JCLI-D-1005033.1.

[30] R. H. Reichle, C. S. Draper, Q. Liu, M. Girotto, S. P. P. Mahanama, R. D. Koster, and G. J. M. De Lannoy, "Assessment of merra-2 land surface hydrology estimates," Journal of Climate, vol. 30, no. 8 , pp. 2937-2960, 2017. DOI: 10.1175/JCLI-D-16-0720.1.

[31] Y. Liu, R. Parinussa, W. Dorigo, R. de Jeu, W. Wagner, A. van Dijk, M. McCabe, and J. Evans, "Developing an improved soil moisture dataset by blending passive and active microwave satellite-based retrievals," Hydrology and Earth System Sciences, vol. 15, Nov. 2010. DOI: 10.5194/hessd-7-6699-2010.

[32] C. Paulik, A. Plocon, S. Hahn, T. Mistelbauer, M. Schmitzer, A Gruber, I. Teubner, and C. Reimer, Tuw-geo/pytesmo: V0.6.10, Apr. 2018. DOI: 10.5281 /zenodo. 1215760.

[33] C. Paulik, W. Preimesberger, M. Schmitzer, and S. Hahn, Tuw geolecmwf_models: V0.5, Jun. 2019. DOI: 10.5281/zenodo.3245388.

[34] H. Hersbach, B. Bell, P. Berrisford, S. Hirahara, A. Horányi, J. Muñoz-Sabater, J. Nicolas, C. Peubey, R. Radu, D. Schepers, A. Simmons, C. Soci, S. Abdalla, X. Abellan, G. Balsamo, P. Bechtold, G. Biavati, J. Bidlot, M. Bonavita, G. De Chiara, P. Dahlgren, D. Dee, M. Diamantakis, R. Dragani, J. Flemming, R. Forbes, M. Fuentes, A. 
Geer, L. Haimberger, S. Healy, R. J. Hogan, E. Hólm, M. Janisková, S. Keeley, P. Laloyaux, P. Lopez, C. Lupu, G. Radnoti, P. de Rosnay, I. Rozum, F. Vamborg, S. Villaume, and J.-N. Thépaut, "The era5 global reanalysis," Quarterly Journal of the Royal Meteorological Society, vol. n/a, no. n/a, DOI: 10.1002/qj.3803.

[35] H. Hersbach, P. de Rosnay, B. Bell, D. Schepers, A. Simmons, C. Soci, S. Abdalla, M. Alonso-Balmaseda, G. Balsamo, P. Bechtold, P. Berrisford, J.-R. Bidlot, E. de Boisséson, M. Bonavita, P. Browne, R. Buizza, P. Dahlgren, D. Dee, R. Dragani, M. Diamantakis, J. Flemming, R. Forbes, A. J. Geer, T. Haiden, E. Hólm, L. Haimberger, R. Hogan, A. Horányi, M. Janiskova, P. Laloyaux, P. Lopez, J. MunozSabater, C. Peubey, R. Radu, D. Richardson, J. Thépaut, F. Vitart, X. Yang, E. Zsótér, and H. Zuo, "Operational global reanalysis: Progress, future directions and synergies with nwp," ECMWF, ERA Report Series 27, Dec. 2018. DOI: 10.21957/tkic6g3wm.

[36] "The era-interim reanalysis: Configuration and performance of the data assimilation system," Quarterly Journal of the Royal Meteorological Society, vol. 137, no. 656, pp. 553-597, 2011. DOI: 10.1002/ qj.828.

[37] G. Balsamo, C. Albergel, A. Beljaars, S. Boussetta, E. Brun, H. Cloke, D. Dee, E. Dutra, J. Muñoz-Sabater, F. Pappenberger, P. de Rosnay, T. Stockdale, and F. Vitart, "Era-interim/land: A global land surface reanalysis data set," Hydrology and Earth System Sciences, vol. 19, no. 1, pp. 389-407, 2015. DOI: 10.5194/hess-19-389-2015.

[38] H. Alexandersson and A. Moberg, "Homogenization of swedish temperature data. part i: Homogeneity test for linear trends," International Journal of Climatology, vol. 17, no. 1, pp. 25-34, 1997. DOI: 10.1002/ (SICI) 1097-0088(199701)17:1〈25::AID-JOC103〉3.0.CO;2-J.

[39] H. B. Mann and D. R. Whitney, "On a test of whether one of two random variables is stochastically larger than the other," The Annals of Mathematical Statistics, vol. 18, no. 1, pp. 50-60, Mar. 1947. DOI: 10.1214/aoms/1177730491.

[40] J. W. Pratt, "Remarks on zeros and ties in the wilcoxon signed rank procedures," Journal of the American Statistical Association, vol. 54, no. 287, pp. 655-667, 1959. DOI: 10.1080/01621459.1959.10501526.

[41] M. Fligner and T. J. Killeen, "Distribution-free two-sample tests for scale," Journal of the American Statistical Association, vol. 71, no. 353 , pp. $210-213$, 1976. DOI: 10.1080/01621459.1976.10481517.

[42] H. Tuomenvirta and H. Alexandersson, "Adjustment of apparent changes in variability of temperature time series," 6th International Meeting on Statistical Climatology, pp. 443-446, 1995.

[43] J. Sheng and F. Zwiers, "An improved scheme for time-dependent boundary conditions in atmospheric general circulation models," Climate Dynamics, vol. 14, no. 7, pp. 609-613, Jun. 1998. DOI: $10.1007 / \mathrm{s} 003820050244$.

[44] P. M. Della-Marta and H. Wanner, "A method of homogenizing the extremes and mean of daily temperature measurements," Journal of Climate, vol. 19, no. 17, pp. 4179-4197, 2006. DOI: $10.1175 /$ JCLI3855.1

[45] J. R. M. Hosking, "L-moments: Analysis and estimation of distributions using linear combinations of order statistics," Journal of the Royal Statistical Society, vol. 52, no. 1, pp. 105-124, 1990.

[46] F. J. Massey Jr., "The kolmogorov-smirnov test for goodness of fit," Journal of the American Statistical Association, vol. 46, no. 253, pp. $68-78,1951$. DOI: 10.1080/01621459.1951.10500769.

[47] W. S. Cleveland, "Robust locally weighted regression and smoothing scatterplots," Journal of the American Statistical Association, vol. 74, no. 368, pp. 829-836, 1979. DOI: 10.1080/01621459.1979.10481038.

[48] S. Seabold and J. Perktold, "Statsmodels: Econometric and statistical modeling with python," in 9th Python in Science Conference, 2010.

[49] X. L. Wang, H. Chen, Y. Wu, Y. Feng, and Q. Pu, "New techniques for the detection and adjustment of shifts in daily precipitation data series," Journal of Applied Meteorology and Climatology, vol. 49, no. 12, pp. 2416-2436, 2010. DOI: 10.1175/2010JAMC2376.1.

[50] L. A. Vincent, X. L. Wang, E. J. Milewska, H. Wan, F. Yang, and V. Swail, "A second generation of homogenized canadian monthly surface air temperature for climate trend analysis," Journal of Geophysical Research: Atmospheres, vol. 117, no. D18, 2012. DOI: 10. 1029/2012JD017859.

[51] W. Dorigo, A. Gruber, R. De Jeu, W. Wagner, T. Stacke, A. Loew, C. Albergel, L. Brocca, D. Chung, R. Parinussa, and R. Kidd, "Evaluation of the esa cci soil moisture product using ground-based observations," Remote Sensing of Environment, vol. 162, pp. 380-395, 2015. DOI: $10.1016 /$ j.rse.2014.07.023.

[52] A. McNally, S. Shukla, K. R. Arsenault, S. Wang, C. D. Peters-Lidard, and J. P. Verdin, "Evaluating esa cci soil moisture in east africa," International Journal of Applied Earth Observation and Geoinformation, vol. 48, pp. 96-109, 2016, Advances in the Validation and Application of Remotely Sensed Soil Moisture - Part 2. DOI: 10.1016/j.jag.2016. 01.001 .

[53] W. Dorigo, R. de Jeu, D. Chung, R. Parinussa, Y. Liu, W. Wagner, and D. Fernández-Prieto, "Evaluating global trends (1988-2010) in harmonized multi-satellite surface soil moisture," Geophysical Research Letters, vol. 39, no. 18, 2012. DOI: 10.1029/2012GL052988.

[54] C. Albergel, W. Dorigo, R. H. Reichle, G. Balsamo, P. de Rosnay, J. Muñoz-Sabater, L. Isaksen, R. de Jeu, and W. Wagner, "Skill and global trend analysis of soil moisture from reanalyses and microwave remote sensing," Journal of Hydrometeorology, vol. 14, no. 4, pp. 1259-1277, 2013. DOI: 10.1175/JHM-D-12-0161.1.

[55] H. Theil, "A rank-invariant method of linear and polynomial regression analysis," in Henri Theils contributions to economics and econometrics, Springer, 1992, pp. 345-381.

[56] H. B. Mann, "Nonparametric tests against trend," Econometrica, vol. 13, no. 3, pp. 245-259, 1945.

[57] M. G. Kendall, "Rank correlation methods.," 1948.

[58] R. O. Gilbert, Statistical methods for environmental pollution monitoring. John Wiley \& Sons, 1987.

[59] H. Caussinus and O. Mestre, "Detection and correction of artificial shifts in climate series," Journal of the Royal Statistical Society: Series C (Applied Statistics), vol. 53, no. 3, pp. 405-425, 2004. DOI: 10.1111/j.1467-9876.2004.05155.x.

[60] F. Zaussinger, W. Dorigo, A. Gruber, A. Tarpanelli, P. Filippucci, and L. Brocca, "Estimating irrigation water use over the contiguous united states by combining satellite and reanalysis soil moisture data," Hydrology and Earth System Sciences, vol. 23, no. 2, pp. 897-923, 2019. DOI: $10.5194 /$ hess-23-897-2019. 


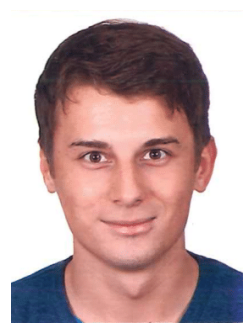

Wolfgang Preimesberger has a B.Sc. and M.Sc. in Geodesy and Geoinformation from Vienna University of Technology (TU Wien). He is currently working in the research group for Climate and Environmental Remote Sensing (CLIMERS) at the department of Geodesy and Geoinformation at TU Wien. As part of the ESA CCI SM and C3S SM team his research focus is on the creation, evaluation and application of long-term satellite soil moisture records.

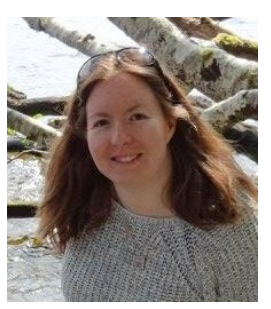

Tracy Scanlon has a B.Sc. in Physics from the University of Bristol and an M.Sc. in Remote Sensing from Cranfield University. She is currently working on the ESA CCI SM and C3S SM products at TU Wien whilst obtaining her $\mathrm{PhD}$ in the quality control remote sensing soil moisture products. Her research interests include the development of improved soil moisture product merging algorithms and the quantification of uncertainties in such products.

Chun-Hsu Su received the B.Sci/B.E and Ph.D. degrees in software engineering and physics from the University of Melbourne, Victoria, Australia in 2006 and 2010, respectively. From 2012-2015, he was with the Department of Infrastructure Engineering, University of Melbourne, to focus on satellite remote sensing and data assimilation. Since 2016, he is a research scientist at the Bureau of Meteorology, with research interests in atmospheric reanalysis and high-resolution climate projection. He remains as a honorary fellow at the University of Melbourne.

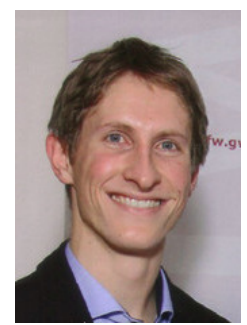

Alexander Gruber was born in Vienna, Austria, in 1988. He received the Dipl.-Ing. (M.Sc.) degree in Geodesy and Geophysics and the Dr.techn. (Ph.D.) degree in Surveying and Geoinformation, both with excellence, from the Vienna University of Technology (TU Wien), Vienna, in 2013 and 2016, respectively. From 2011 until 2017 he has been a researcher and a lecturer at the Department of Geodesy and Geoinformation of TU Wien. Since 2018 he has been a postdoctoral researcher at the Katholieke Universiteit Leuven (KU Leuven), Belgium, and since 2019 he is a senior postdoctoral fellow of the Research Foundation Flanders (FWO). His main research interests include geophysical parameter retrieval from earth observation data, earth system modelling, and data assimilation. Dr. Gruber has authored or co-authored a large number of peer-reviewed journal papers, conference papers, and book chapters and is a reviewer for many scientific journals in the field of remote sensing and earth observation.

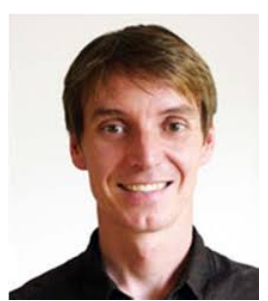

Wouter Dorigo received the M.Sc. degree in physical geography with an emphasis on remote sensing from Utrecht University, Utrecht, The Netherlands, in 2000, and the Ph.D. degree in remote sensing from the Technical University of Munich, Munich, Germany, in 2008. From 2002 to 2006, he was with the Imaging Spectroscopy Group, German Remote Sensing Data Center, German Aerospace Center, Oberpfaffenhofen, Germany. Since 2007, he has been a Researcher, a Lecturer, and a Scientific Project Coordinator with the Research Group Remote Sensing, Department of Geodesy and Geoinformation, Vienna University of Technology (TU Wien), Vienna, Austria, where he is currently a Professor in climate and environmental remote sensing. He has authored or co-authored more than 60 publications in refereed journal papers and conference proceedings. Using earth observation data, he tries to understand and quantify the dynamics and interactions of vegetation and the water cycle in a changing climate. His research interests include remote sensing of soil moisture and vegetation, with a focus on geophysical parameter retrieval through physical and semiempirical methods, calibration and validation, process automation, up and downscaling, image classification, data merging, and time series analysis Dr. Dorigo was a recipient of the 2015 Science Award of TU Wien for the water cycle in a changing climate. 


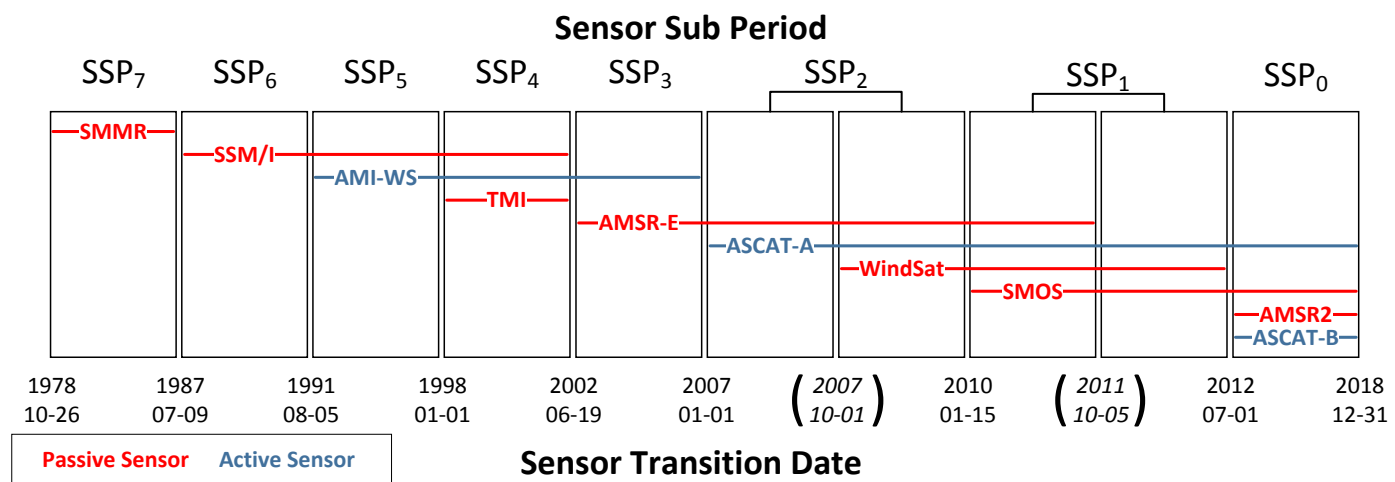

Fig. 1: ESA CCI SM v04.5 COMBINED sensor periods: Horizontal lines describe the temporal coverage of active (red) and passive (blue) sensor SM products. Boxes indicate changes in the set of merged sensor products. Sensor transition dates accord to the dates of potential breaks in the data set and define the Sensor Sub-Periods (SSP). $S S P_{0}$ to $S S P_{7}$ therefore describe the minimal assumed homogeneous sub-periods in the ESA CCI SM product. Two transitions are excluded in this study (parenthesised dates) to avoid sub-periods that are shorter than one year.
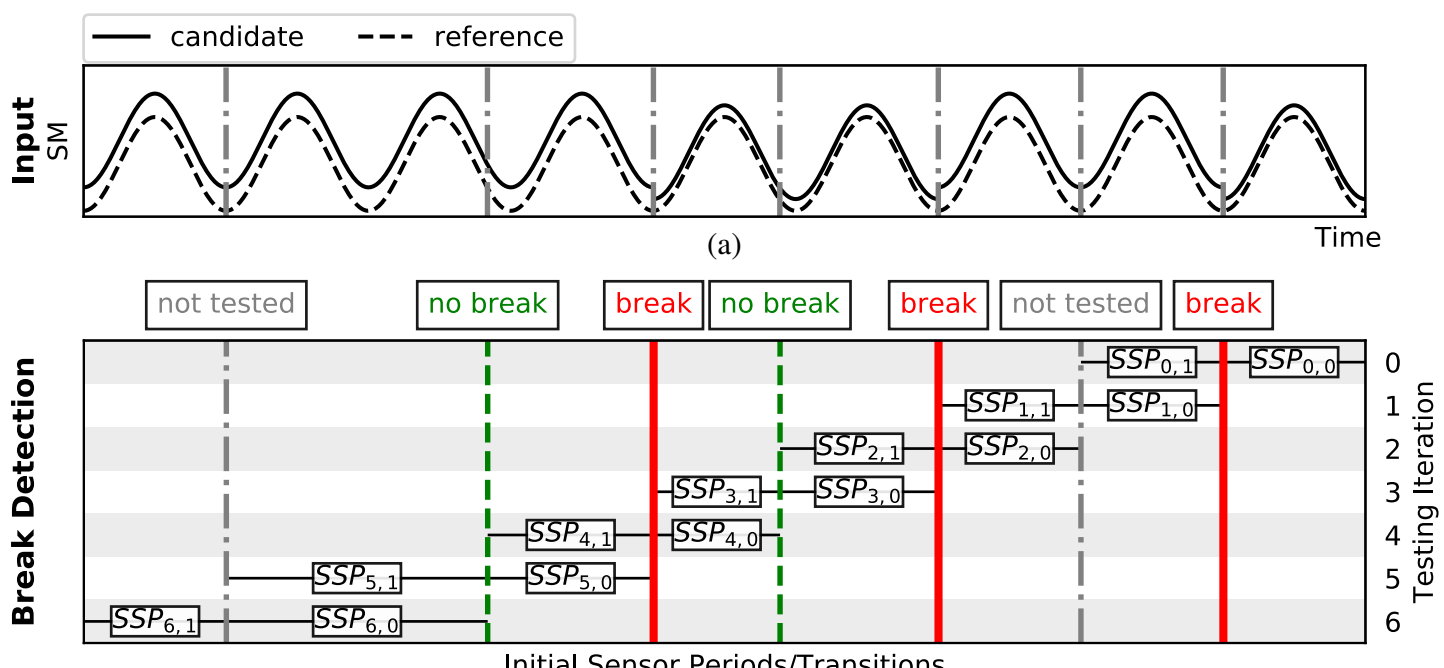

(b)

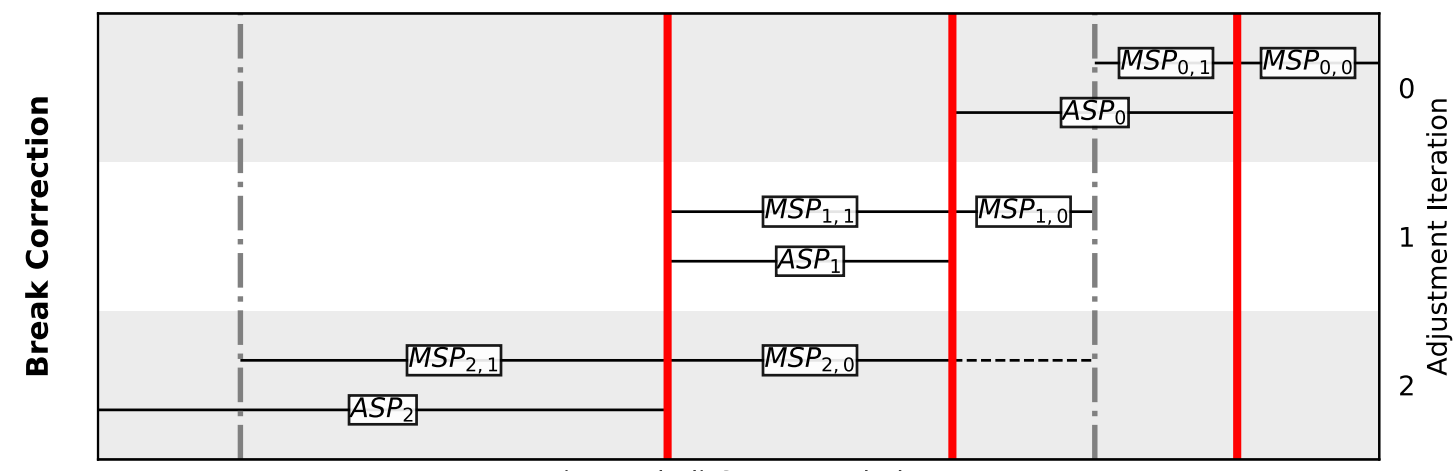

(Extended) Sensor Periods

(c)

Fig. 2: Schematic representation of period nomination for break detection (b) and correction (c) and concept of period extension for correction. The original sensor transition dates in each candidate series (vertical lines in the top segment) are initially tested as shown in the middle segment: Testing detects homogeneities (dashed green lines) and breaks (solid red lines) as well as un-testable transitions (dashed grey lines). The lower segment shows the period extension for adjustment based on the initial break detection, where model sub-periods (MSPs) for quantifying the break are extended across homogeneities (green) only. Due to untested transition dates, adjustment sub-periods (ASPs) - periods that corrections derived from MSPs are applied to cannot be equal to the respective MSPs and are therefore defined as the candidate observations between detected breaks. 


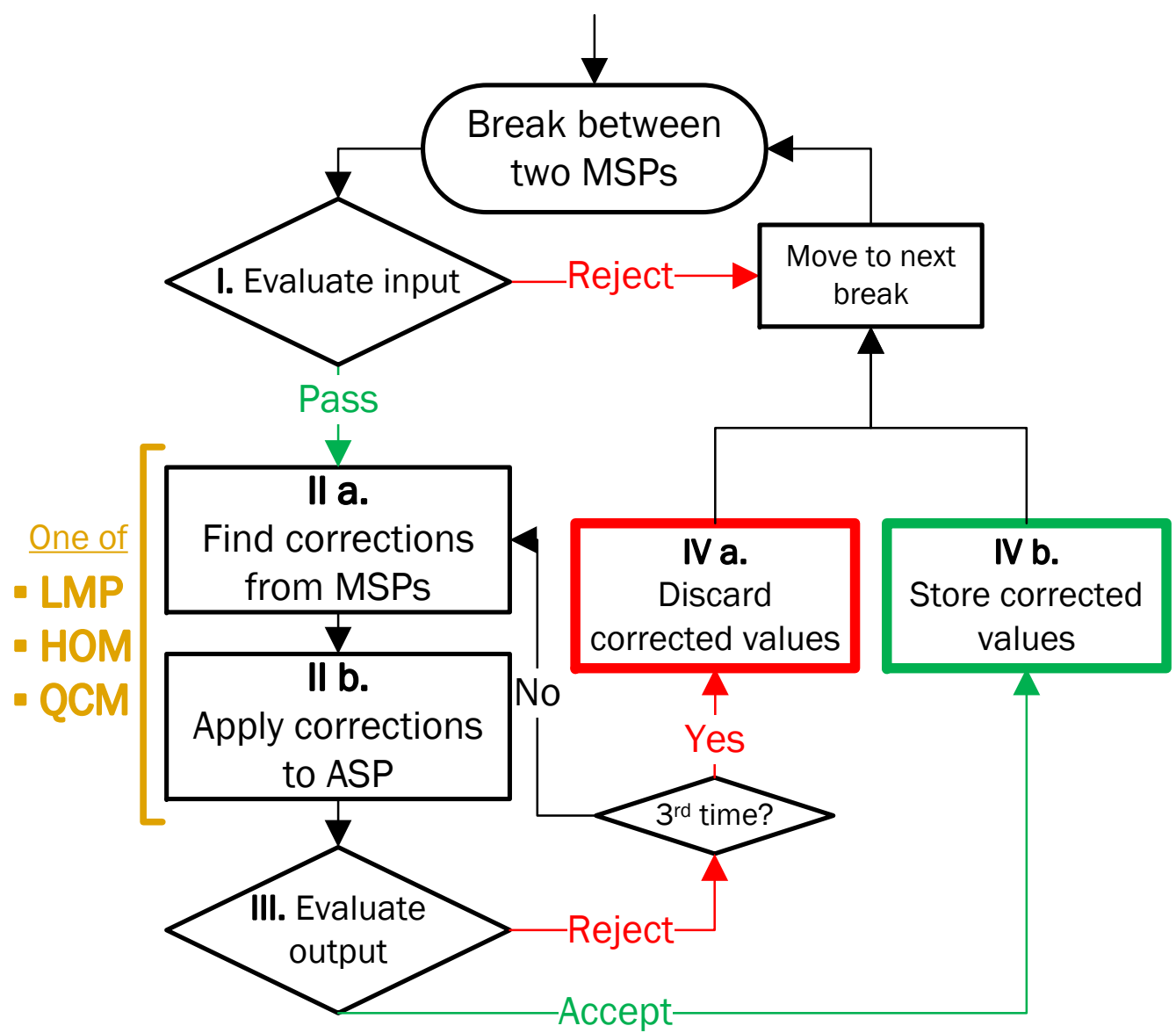

Fig. 3: Process cycle of the iterative correction between detected breaks.

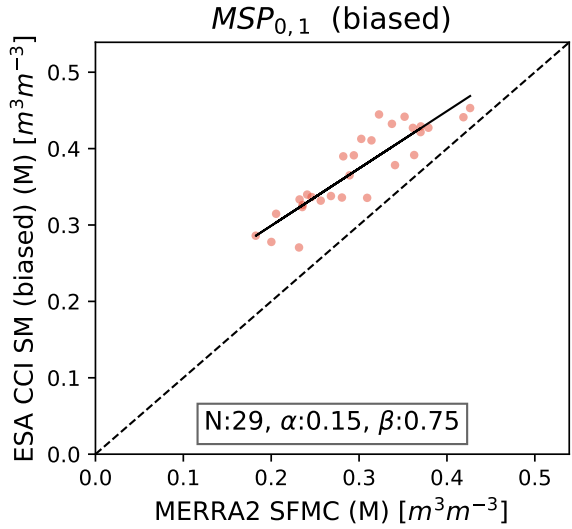

(a)

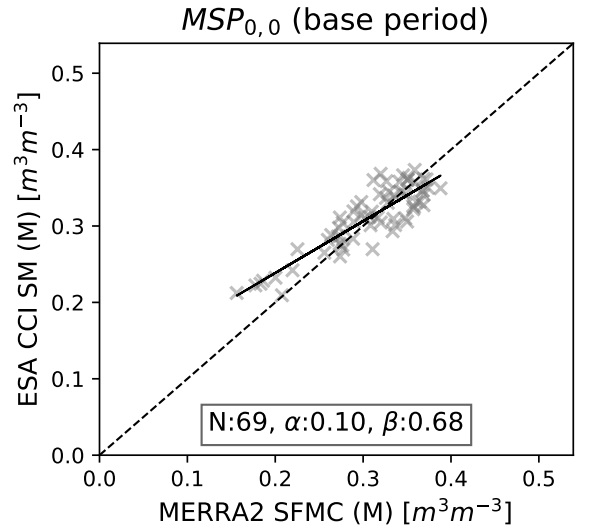

(b)

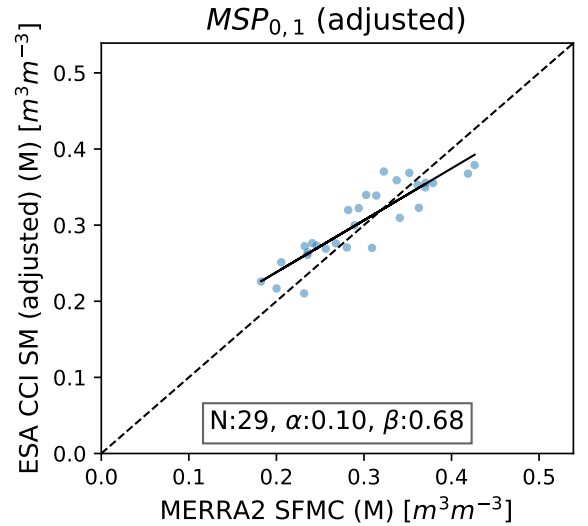

(c)

Fig. 4: LMP adjustment - linear regression models for the two MSPs, i.e. before (a) and after (b) the detected break. The introduced bias leads to a discrepancy in the models coefficients. For comparison (c) shows the same period as (a), but after successful correction, which resulted in matching the model parameters to (b). 


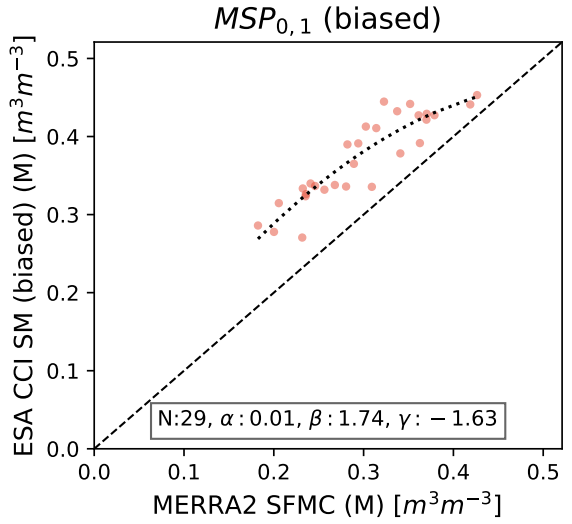

(a)

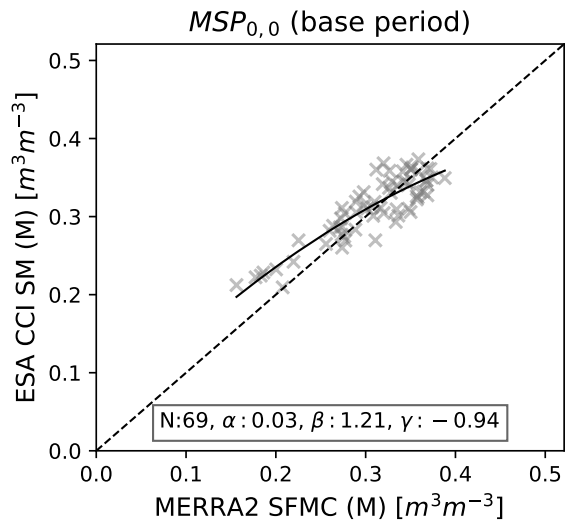

(b)

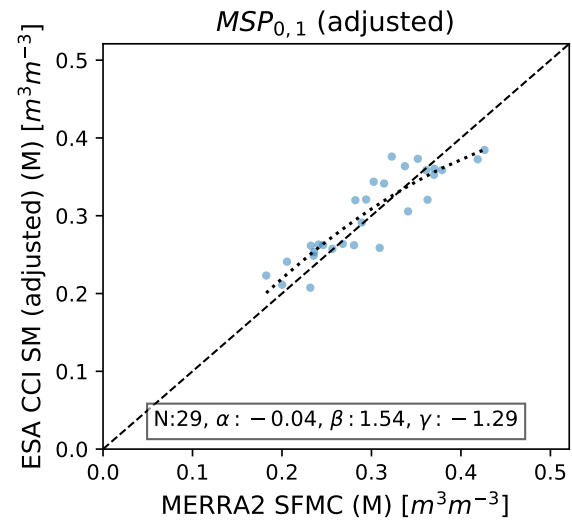

(c)

Fig. 5: HOM adjustment - Second order polynomial regression model for the reference period of the same example series as in Section III-B2 from monthly resampled values after the introduced break (b). The model is used to create homogeneous predictions to find corrections for the biased candidate observations (a) and subsequently adjust them (c). The dotted lines in (a) and (c) indicate that the regressions are only shown for comparison and not used for correction.

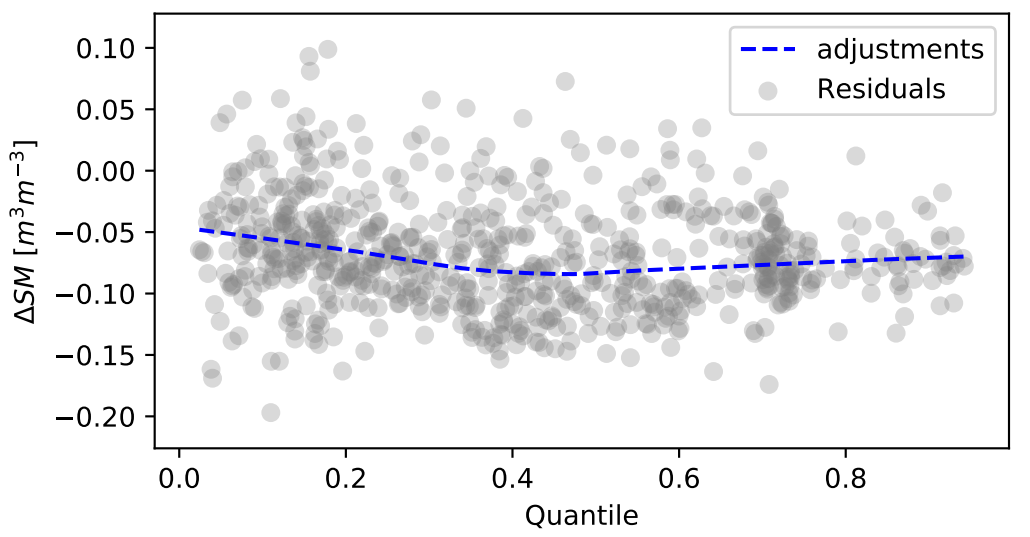

Fig. 6: SM residuals between observed and predicted candidate (circles) and derived quantile adjustments (LOWESS fit with $\alpha=0.6$, dashed blue line) within the period $M S P_{0,1}$ of the example time series.

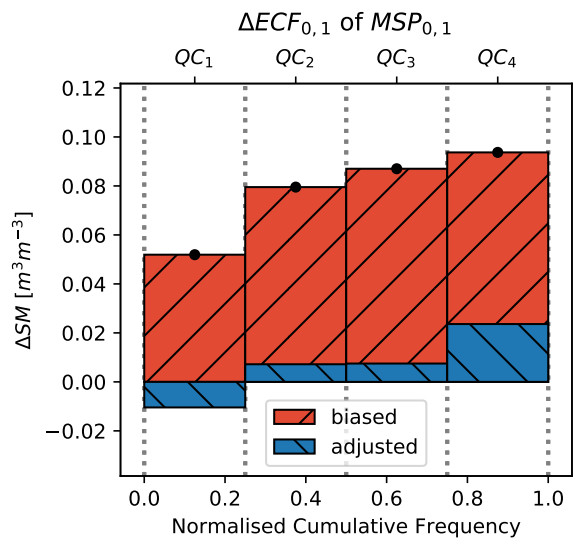

(a)

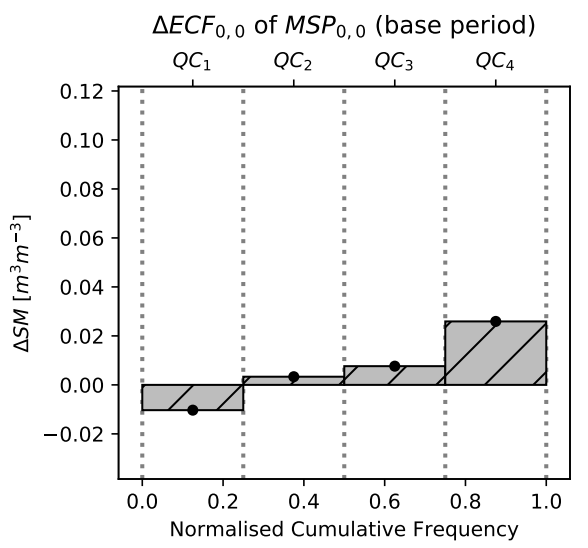

(b)

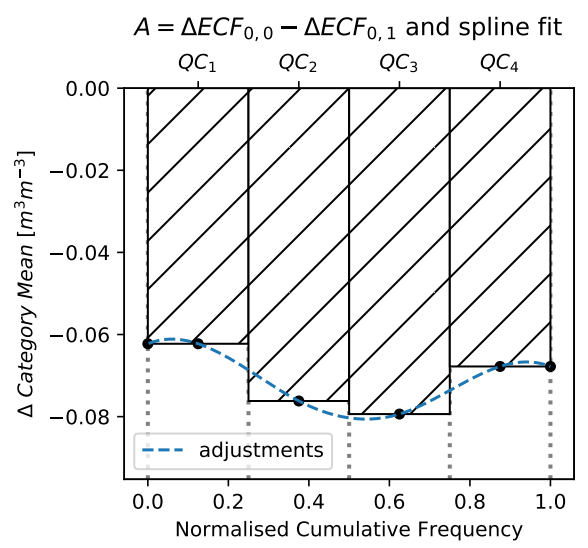

(c)

Fig. 7: QCM adjustment - $\triangle E C F$ for the MSP before - (a), red bars - and after (b) the transition date of the introduced break. Differences between $\triangle E C F s$ (c) are used to find adjustments for all biased observations (spline interpolation, dashed blue line), and result in matching the ECF of the candidate in $M S P_{0,1}$ to the reference relative to differences in $M S P_{0,0}$ after correction - (a), blue bars. 
2012-07-01

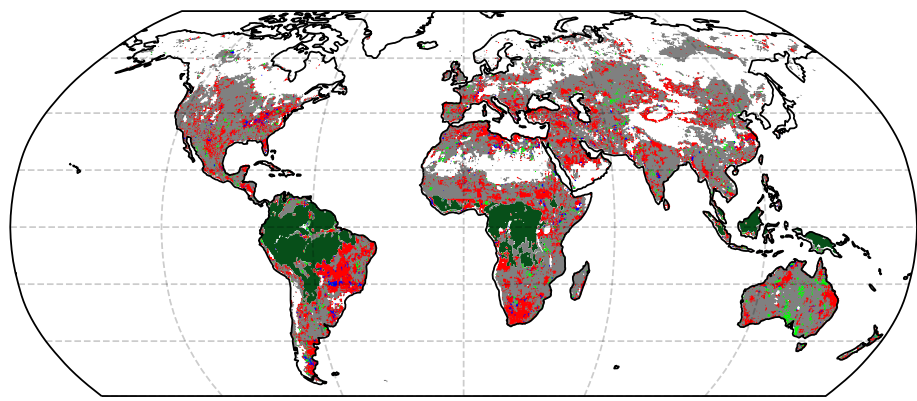

(a) 2012-07-01: Before adjustment

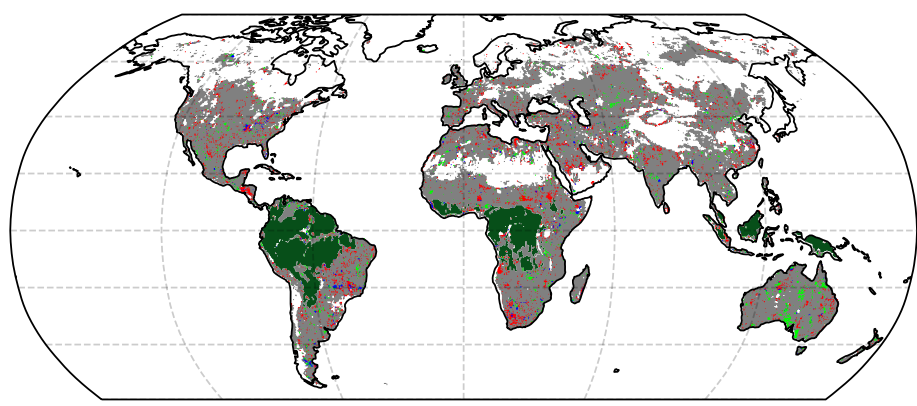

(c) 2012-07-01: After LMP adjustment

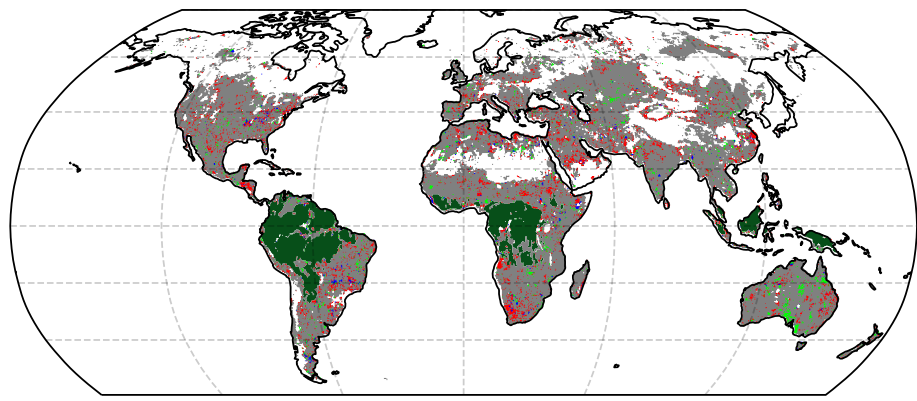

(e) 2012-07-01: After HOM adjustment

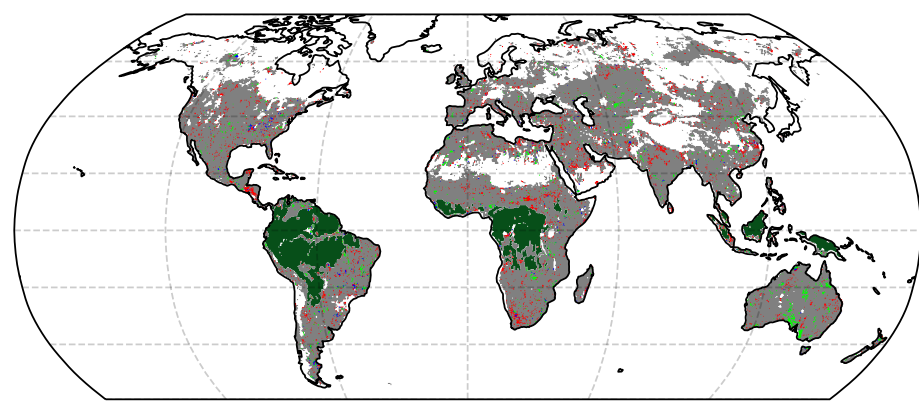

(g) 2012-07-01: After QCM adjustment
2002-06-19

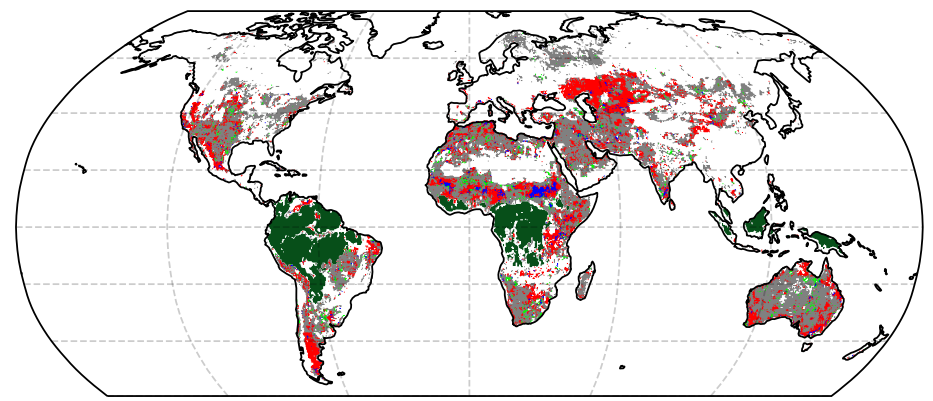

(b) 2002-06-19: Before adjustment

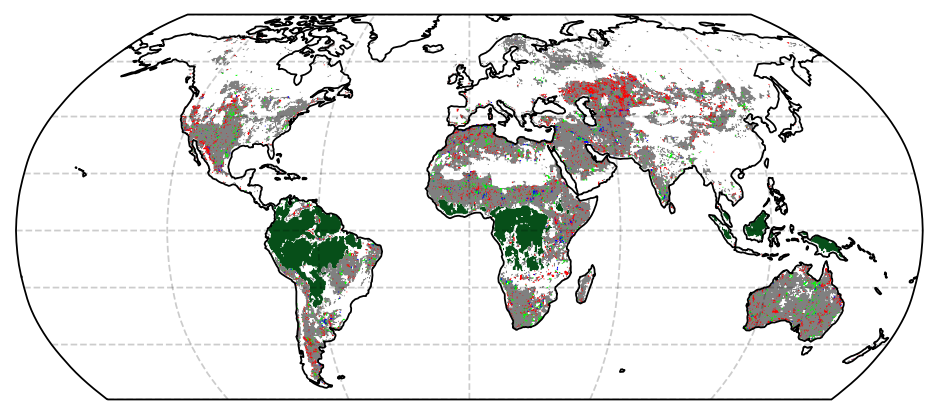

(d) 2002-06-19: After LMP adjustment

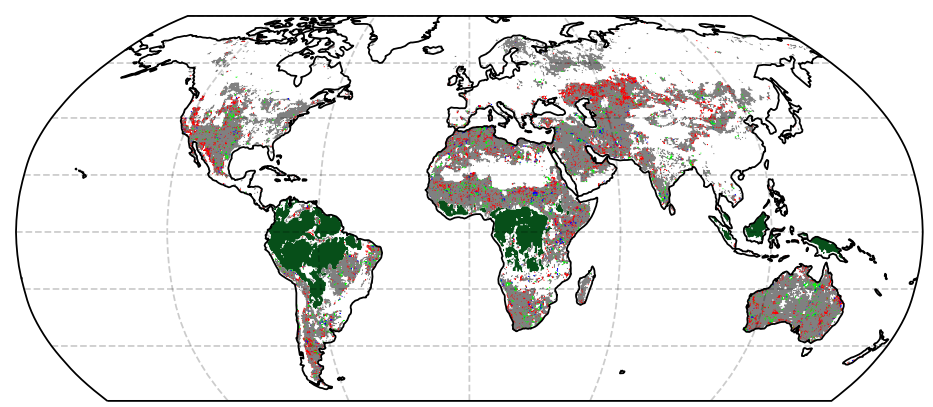

(f) 2002-06-19: After HOM adjustment

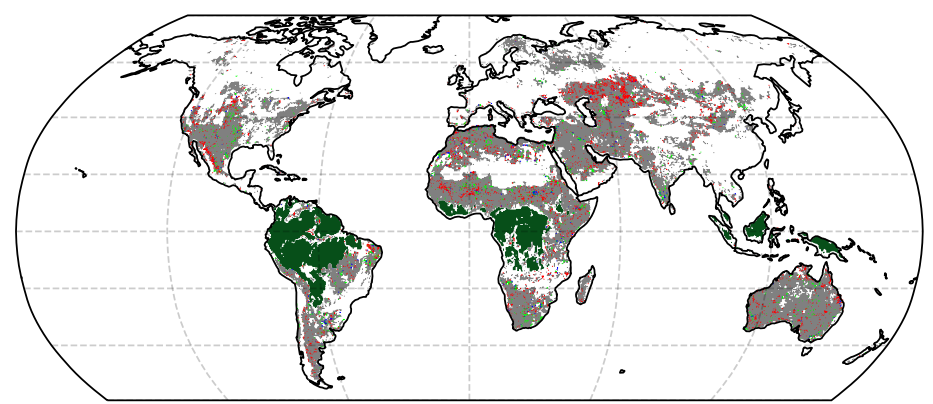

(h) 2002-06-19: After QCM adjustment

\section{Wo Break Mean Vartance Both Wot Vested Masked}

Fig. 8: Break detection for ESA CCI SM before adjustment (a, b), after adjustment with LMP (c, d), after adjustment with HOM $(\mathrm{e}, \mathrm{f})$ and after adjustment with QCM $(\mathrm{g}, \mathrm{h}$ ) for two example sensor transition dates: 2012-07-01 (left column) and 2002-06-19 (right column). Plots for the remaining transition dates can be found in SI Figs. 7, 12, 17, and 21. Red areas indicate a break in mean (WK). Light Green areas indicate a shift in variance (FK). Dark blue areas define points where both tests were positive and grey areas where no (significant) break in mean and variance was detected. White areas indicate that testing was not performed because prerequisites are not met (see Section III-A and SI Fig. 8). Dark green areas are excluded according to the ESA CCI SM rainforest mask. 


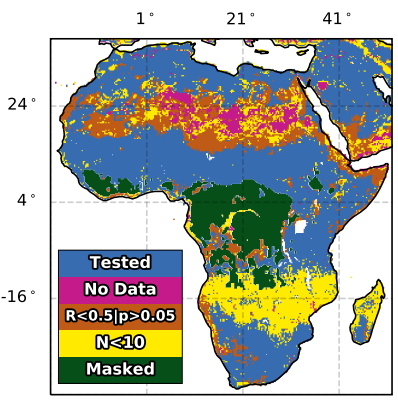

(a) Break detection evaluation

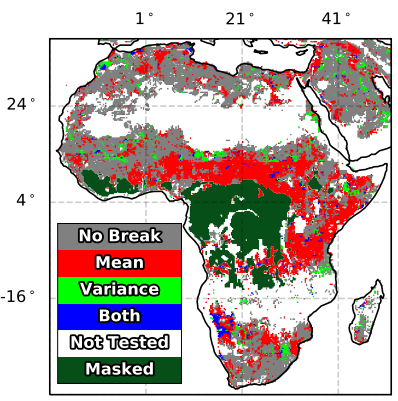

(b) Detection - Before Adjustment

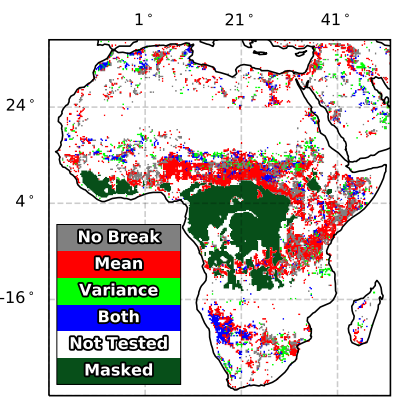

(c) Detection - Extended Periods

Fig. 9: 1998-01-01 - subset of global results. Evaluation before initial testing (a) and detected breaks using (unextended) SSPs (b). Detected breaks after SSP extension (MSPs) (c) are the basis for break correction (also see SI Figs. 9, 13 and 18). Masked areas due to dense vegetation are excluded according to the previous figures.

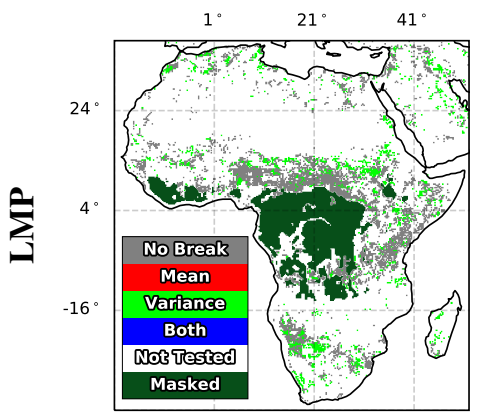

(a)

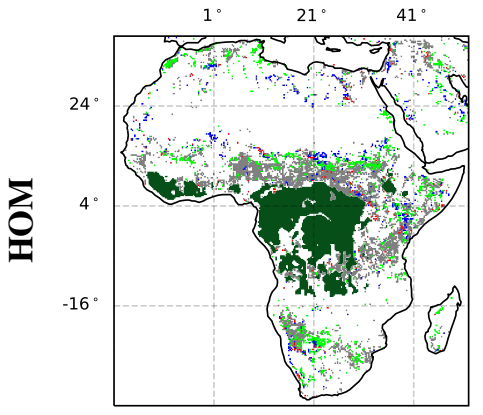

(d)

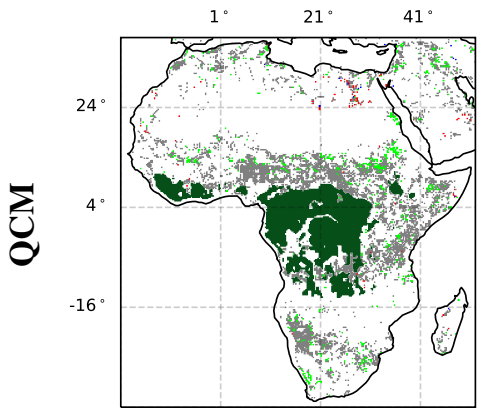

(g)

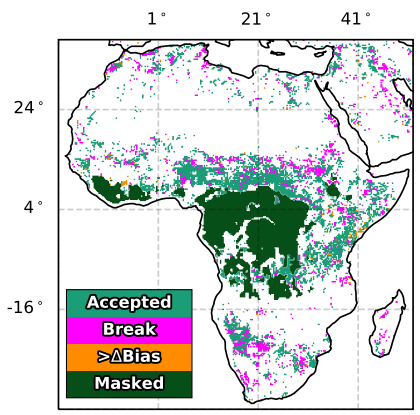

(b)

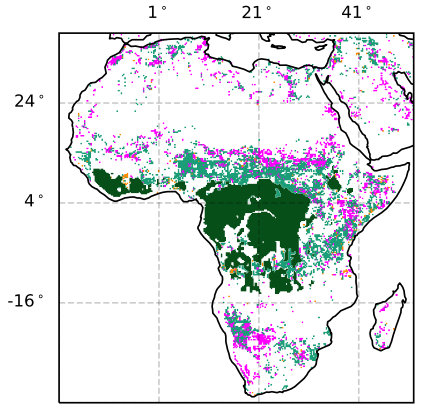

(e)

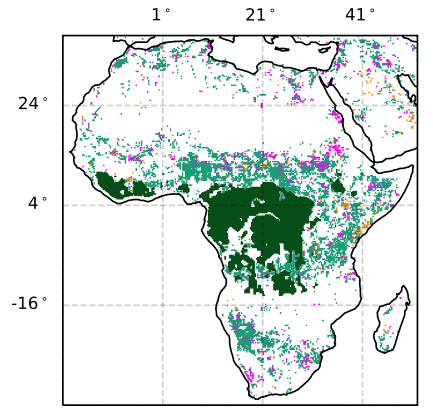

(h)

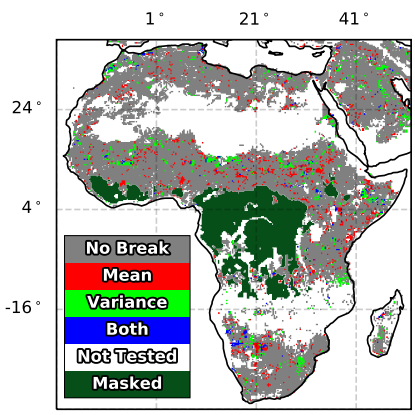

(c)

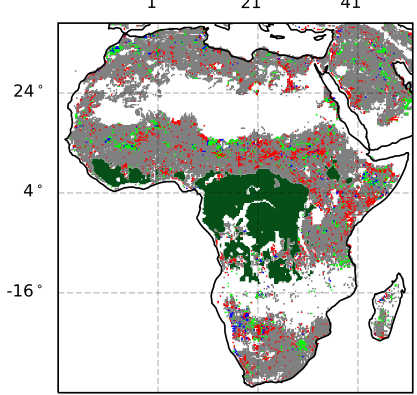

(f)

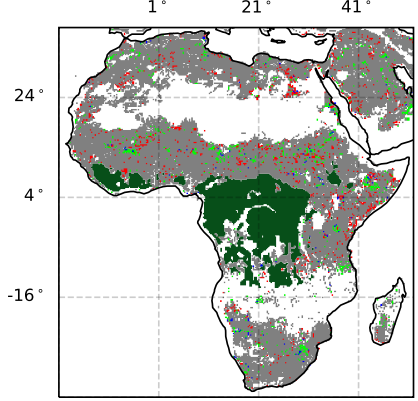

(i)

Fig. 10: 1998-01-01 - Test results between MSPs after applying corrections (left column), adjustment output evaluations (middle column), and detected breaks in a separate repetition of testing between SSPs (right column). Results are shown for each of the three methods: LMP (a-c), HOM (d-f) and QCM (g-i). Densely vegetated areas are masked out as in previous plots. For the according global plots for the other transition dates, see SI Figs. 10, 11 and 15, 16 and 19, 20. 


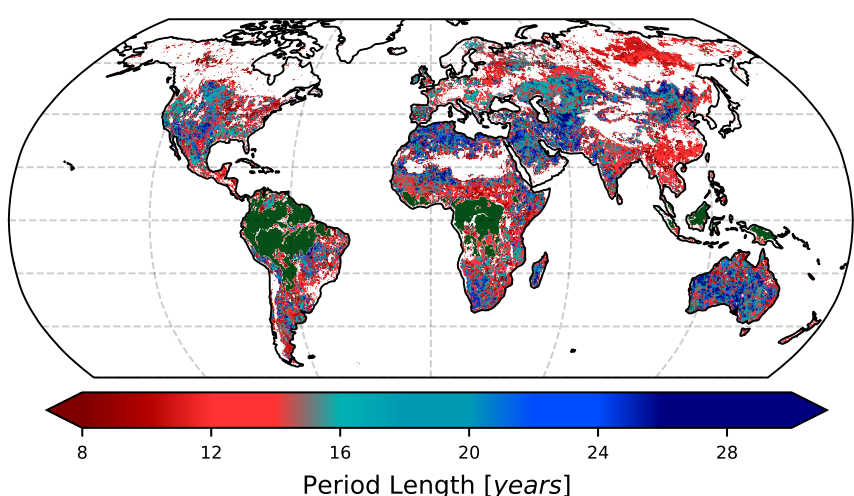

Fig. 11: Length of the longest homogeneous sub-period in the original, unadjusted ESA CCI SM data set. As before, green areas indicate masking due to dense vegetation. The according plots for period lengths after correction are in SI Fig. 22.

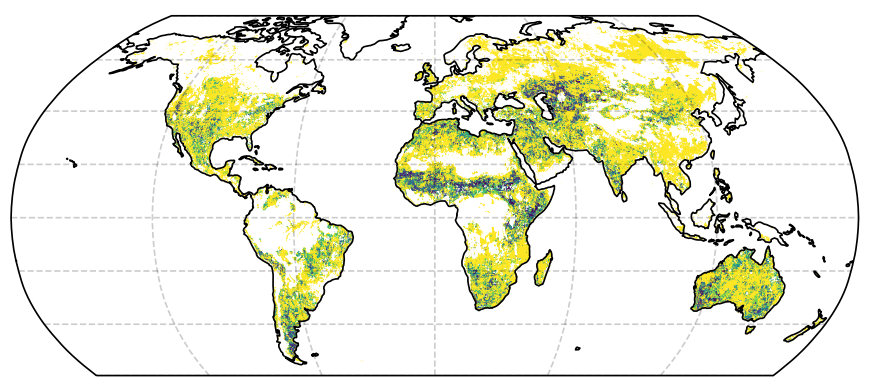

(a)

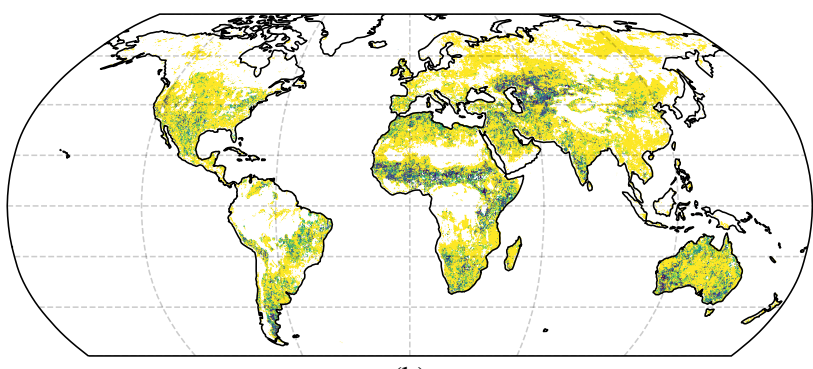

(b)

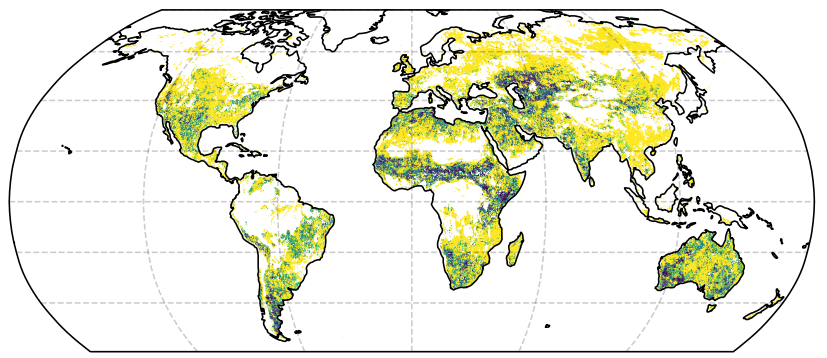

(c)

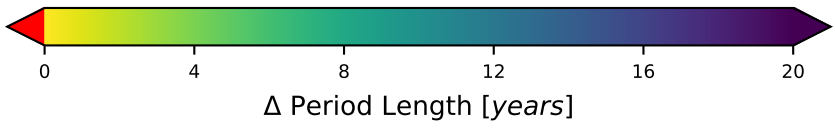

Fig. 12: Increase in length of the longest homogeneous period after correction with LMP (a), HOM (b) and QCM (c).

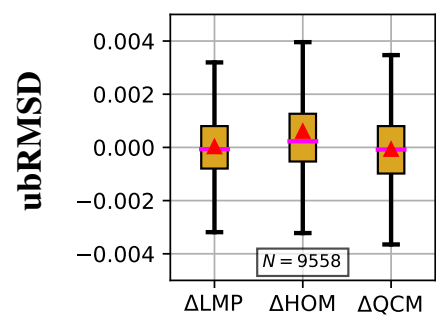

(a) Cropland

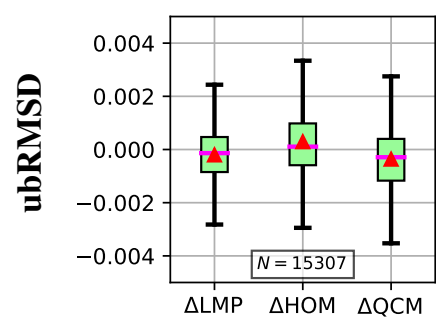

(c) Grassland

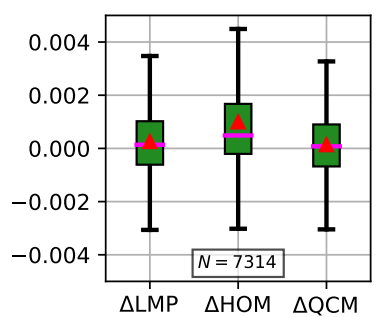

(b) Tree Cover

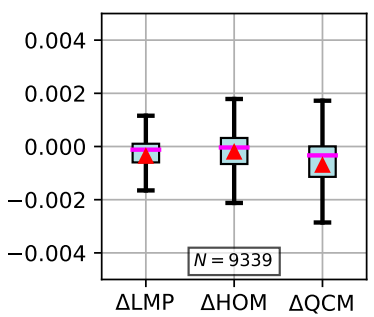

(d) Other
Fig. 13: Difference in ubRMSD between the unadjusted and adjusted versions of ESA CCI SM and ERA5. Results are binned based on ESA CCI Land Cover information for the year 2010 as follows: Cropland (LC classes 10-30), Tree Cover (LC classes 40-90, 160, 170), Grassland (LC classes 120-130, 180) and all remaining points (Other). $\Delta$ indicates the mean of all values, - the median, box edges represent the quartiles and whiskers $1.5 * I Q R$.

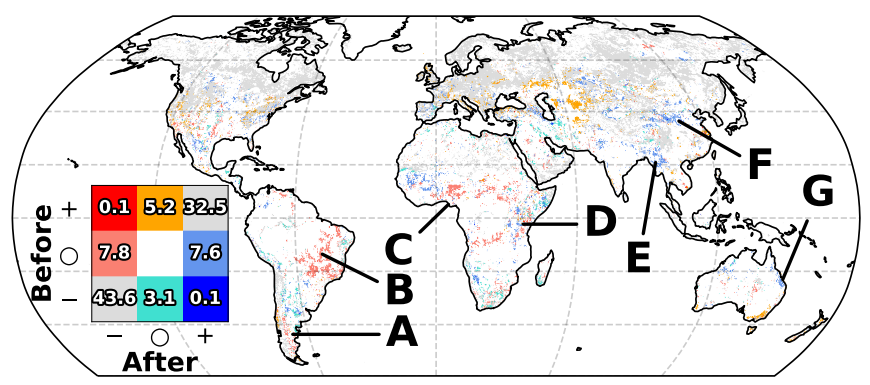

Fig. 14: Sign change in significant $(p<0.05)$ SM trends after QCM adjustment. "+" indicates wetting, "-" drying and " $\bigcirc$ " non-significant trends before, respectively after adjustment. Inset numbers are in $\%$ of significant trends. 


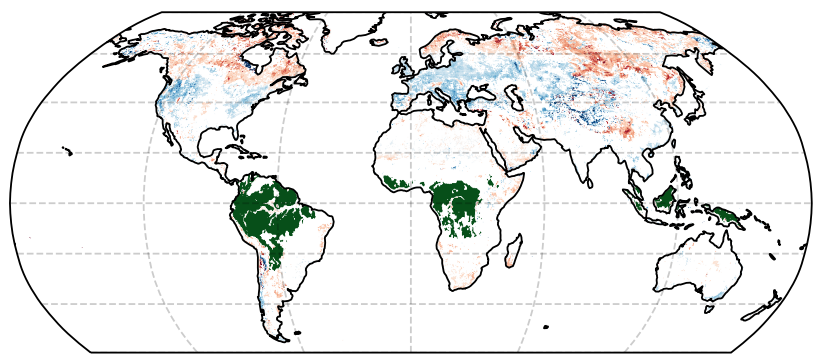

(a) ESA CCI SM

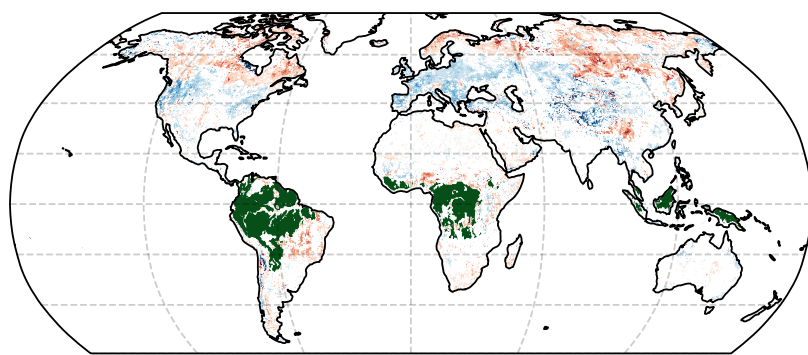

(b) ESA CCI SM (QCM)

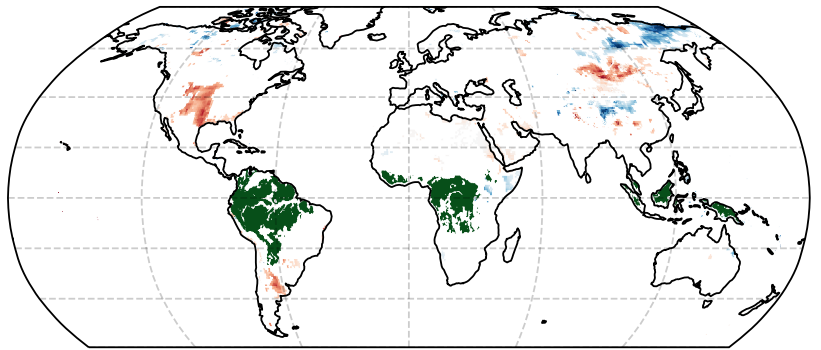

(c) ERA-Interim/LAND

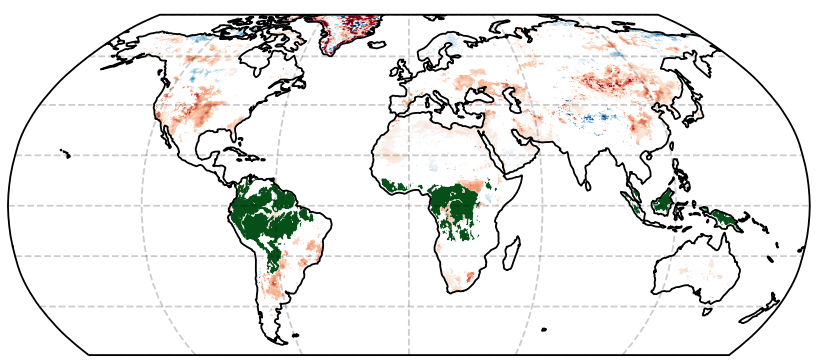

(d) ERA5

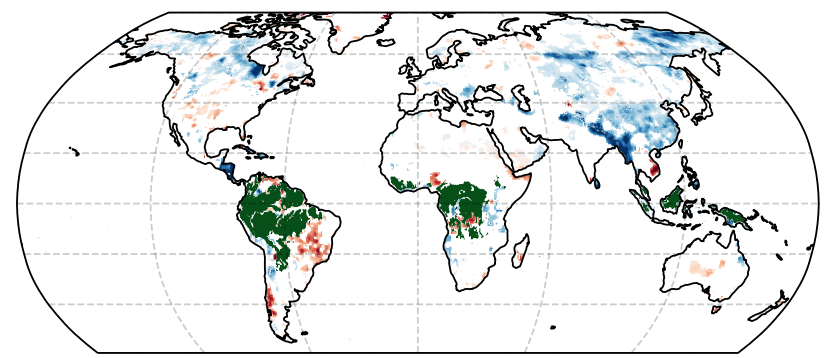

(e) MERRA2

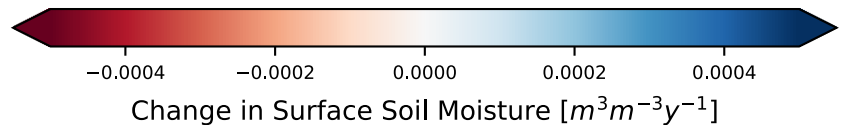

Fig. 15: Significant SM trends: In the original (a) and QCM adjusted (b) ESA CCI SM data, in ERA-Interim/LAND SWVL1 (c), ERA5 SWVL1 (d) and MERRA2 SFMC (e).
TABLE I

TEST STATISTICS BEFORE (ORIG.) AND AFTER ADJUSTMENT WITH LMP, HOM AND QCM

\begin{tabular}{|c|c|c|c|c|c|c|}
\hline & \multirow{2}{*}{$\begin{array}{c}\text { Test }^{* *} \\
\text { Coverage }\end{array}$} & \multicolumn{4}{|c|}{ Break Detection } \\
\hline & & & Mean* & Variance $^{*}$ & Both $^{*}$ & No Break* \\
\hline \multirow{4}{*}{ 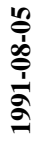 } & orig. & 20.17 & 17.84 & $\underline{4.40}$ & 1.61 & 76.15 \\
\hline & LMP & 20.14 & 5.30 & 3.75 & 0.94 & 90.01 \\
\hline & $\mathrm{HOM}$ & 20.13 & 6.13 & 3.80 & 1.13 & 88.94 \\
\hline & QCM & 20.16 & 4.39 & 3.65 & 0.66 & 91.30 \\
\hline \multirow{4}{*}{ 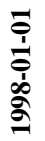 } & orig. & 34.22 & 20.53 & 3.53 & 1.52 & 74.41 \\
\hline & LMP & 34.15 & 5.10 & 3.37 & 0.92 & 90.61 \\
\hline & $\mathrm{HOM}$ & 34.13 & 7.09 & 3.40 & 0.98 & 88.52 \\
\hline & $\mathrm{QCM}$ & 34.18 & 3.87 & 2.81 & 0.52 & 92.80 \\
\hline \multirow{4}{*}{ 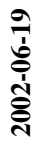 } & orig. & 29.34 & $\underline{29.13}$ & 3.85 & $\underline{3.19}$ & 63.83 \\
\hline & LMP & 29.31 & 9.54 & 4.16 & 1.45 & 84.85 \\
\hline & HOM & 29.28 & 11.32 & 4.00 & 1.39 & 83.30 \\
\hline & $\mathrm{QCM}$ & 29.33 & 8.34 & 3.13 & 0.90 & 87.63 \\
\hline \multirow{4}{*}{ 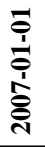 } & orig. & 39.13 & 24.02 & 3.58 & 2.79 & 69.61 \\
\hline & LMP & 39.09 & 8.81 & 3.73 & 1.76 & 85.70 \\
\hline & HOM & 39.08 & 10.64 & 3.74 & 1.67 & 83.95 \\
\hline & QCM & 39.11 & 7.38 & 3.00 & 1.06 & 88.55 \\
\hline \multirow{4}{*}{$\begin{array}{l}\frac{n}{1} \\
\frac{1}{0} \\
\stackrel{1}{0} \\
\frac{d}{8}\end{array}$} & orig. & 53.08 & 12.97 & 1.83 & 0.55 & 84.65 \\
\hline & LMP & 53.04 & 4.71 & 1.70 & 0.43 & 93.17 \\
\hline & $\mathrm{HOM}$ & 53.03 & 5.14 & 1.68 & 0.37 & 92.81 \\
\hline & QCM & 53.07 & 3.86 & 1.26 & 0.25 & 94.63 \\
\hline \multirow{4}{*}{ 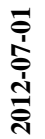 } & orig. & 51.49 & 25.07 & 3.13 & 1.93 & 69.87 \\
\hline & LMP & 51.48 & 7.25 & 3.23 & 1.15 & 88.37 \\
\hline & $\mathrm{HOM}$ & 51.47 & 9.12 & 3.20 & 1.08 & 86.59 \\
\hline & QCM & 51.48 & 5.64 & 2.52 & 0.67 & 91.17 \\
\hline
\end{tabular}

${ }^{* *}$ in \% of 229392 ESA CCI SM land points without dense vegetation. $*$ in $\%$ of tested points

Underlined numbers indicate the highest numbers of relative breaks of each type across all transition dates, bold numbers indicate the best performing method in terms of reduced breaks for all transition dates. Red numbers indicate an increase in breaks compared to the un-adjusted observations.

TABLE II

GLOBAL STATISTICS FOR ADJUSTMENT COVERAGE

\begin{tabular}{llcccc}
\hline & & Accepted* $^{*}$ & Break* $^{*}$ & UnCorr.* $^{*}$ & $>\Delta$ Bias* $^{*}$ \\
\hline \hline $\mathbf{1 9 9 1 - 0 8 - 0 5}$ & LMP & 61.50 & 22.92 & 3.52 & 12.05 \\
& HOM & 59.59 & 27.48 & 1.17 & 11.76 \\
& QCM & 69.77 & 12.23 & 3.52 & 14.48 \\
\hline $\mathbf{1 9 9 8 - 0 1 - 0 1}$ & LMP & 66.79 & 26.68 & 3.22 & 3.31 \\
& HOM & 60.29 & 33.09 & 1.76 & 4.85 \\
& QCM & 78.17 & 14.74 & 3.24 & 3.86 \\
\hline $\mathbf{2 0 0 2 - 0 6 - 1 9}$ & LMP & 65.75 & 28.45 & 2.43 & 3.36 \\
& HOM & 64.33 & 31.08 & 0.91 & 3.68 \\
& QCM & 77.24 & 16.84 & 2.43 & 3.48 \\
\hline $\mathbf{2 0 0 7 - 0 1 - 0 1}$ & LMP & 59.30 & 31.30 & 2.19 & 7.21 \\
& HOM & 55.23 & 35.84 & 1.26 & 7.67 \\
& QCM & 71.77 & 17.16 & 2.18 & 8.89 \\
\hline $\mathbf{2 0 1 0 - 0 1 - 1 5}$ & LMP & 61.80 & 25.84 & 2.11 & 10.25 \\
& HOM & 61.78 & 26.39 & 0.98 & 10.85 \\
& QCM & 75.10 & 10.06 & 2.11 & 12.73 \\
\hline $\mathbf{2 0 1 2 - 0 7 - 0 1}$ & LMP & 72.50 & 20.65 & 2.20 & 4.65 \\
& HOM & 69.10 & 24.44 & 0.87 & 5.58 \\
& QCM & 82.35 & 9.60 & 2.20 & 5.85 \\
\hline
\end{tabular}

$*$ in $\%$ of detected breaks 